\title{
Silicon-Integrated Telecommunications Photon-Spin Interface
}

\author{
L. Bergeron $\odot,{ }^{1}$ C. Chartrand, ${ }^{1}$ A. T. K. Kurkjian, ${ }^{1}$ K. J. Morse, ${ }^{1}$ H. Riemann, ${ }^{2}$ N. V. Abrosimov, ${ }^{2}$ \\ P. Becker, ${ }^{3}$ H.-J. Pohl,${ }^{4}$ M. L. W. Thewalt $\odot,{ }^{1}$ and S. Simmons ${ }^{1, *}$ \\ ${ }^{1}$ Department of Physics, Simon Fraser University, Burnaby, British Columbia, V5A 1S6 Canada \\ ${ }^{2}$ Leibniz-Institut für Kristallzüchtung, Berlin 12489, Germany \\ ${ }^{3}$ Physikalisch-Technische Bundestanstalt Braunschweig, Braunschweig 38116, Germany \\ ${ }^{4}$ VITCON Projectconsult GmbH, Jena 07743, Germany
}

(Received 15 June 2020; revised 18 August 2020; accepted 10 September 2020; published 2 October 2020)

\begin{abstract}
Long-distance entanglement distribution is a vital capability for quantum technologies. An outstanding practical milestone towards this aim is the identification of a suitable matter-photon interface that possesses, simultaneously, long coherence lifetimes and efficient telecommunication-band optical access. In this work we report upon the $T$ center, a silicon defect with long-lived spins and spin-selective bound exciton optical transitions at $1326 \mathrm{~nm}$ in the telecommunications $O$-band. In this first study of $T$ centers in ${ }^{28} \mathrm{Si}$, we present the temperature dependence of the zero-phonon line, report ensemble zero-phonon linewidths as narrow as 33(2) MHz, and elucidate the excited state spectrum of the bound exciton. Magnetophotoluminescence, in conjunction with magnetic resonance, is used to observe twelve distinct orientational subsets of the $T$ center, which are independently addressable due to the anisotropic $g$ factor of the bound exciton's hole spin. Here we show that the $T$ center in ${ }^{28} \mathrm{Si}$ offers electron and nuclear spin lifetimes beyond a millisecond and second, respectively, as well as optical lifetimes of 0.94(1) $\mu$ s and a Debye-Waller factor of 0.23(1). This work represents a significant step towards coherent photonic interconnects between longlived silicon spins, spin-entangled telecom single-photon emitters, and spin-dependent silicon-integrated photonic nonlinearities for future global quantum technologies.
\end{abstract}

DOI: 10.1103/PRXQuantum.1.020301

\section{INTRODUCTION}

The global search for a high-performance quantum interface between telecom photons and long-lived matter qubits is ongoing [1]. The predominant photon-spin candidates presently under study either do not operate at telecom wavelengths or are not hosted within silicon. Technologies such as frequency conversion [2] or evanescent integration with silicon photonics $[3,4]$ are being developed to address the individual shortcomings of such interfaces.

Silicon is a convenient and attractive host for a photonspin interface as it underpins both the most established integrated electronics and integrated photonics platforms. Silicon, and in particular isotopically purified ${ }^{28} \mathrm{Si}$, is a host to many atomically reproducible defects with exceptional spin and/or optical properties. Phosphorus donors possess ultra-long spin lifetimes of up to $3 \mathrm{~h} \mathrm{[5]} \mathrm{but} \mathrm{do}$ not interact strongly with light. Singly-ionized chalcogen

\footnotetext{
*s.simmons@sfu.ca
}

Published by the American Physical Society under the terms of the Creative Commons Attribution 4.0 International license. Further distribution of this work must maintain attribution to the author(s) and the published article's title, journal citation, and DOI. donors, such as ${ }^{77} \mathrm{Se}^{+}$, offer both long-lived spins and relatively strong interactions with light [6,7], however the wavelengths involved are in the technically challenging mid-infrared. Erbium defects in silicon [8,9] offer weak dipole-forbidden telecom optical transitions and potentially long-lived spins [10], yet complex readily with other silicon defects into a wide selection of symmetry sites and complexes, only a small fraction of which are optically active [11].

The family of silicon defects known as radiation damage centers, including the well studied $G, C$, and $W$ centers, have been the subject of a number of recent studies [12-16] due to their bright luminescence, submicrosecond luminescence lifetimes, near radiatively limited optical linewidths in ${ }^{28} \mathrm{Si}$, and wavelengths in the telecommunication bands. However, despite early reports that the $G$ center in particular is connected to an optically detected magnetic resonance (ODMR) signal [17], the dominant zero-phonon line (ZPL) transitions of the $G$, as well as the $C$ and $W$ radiation damage centers, have each been shown to be singlet-tosinglet transitions with no ground state unpaired electron or hole spins in which to hold quantum information.

A few less well-studied radiation damage centers, in particular the $T$ [18-31], I [24-27], and $M$ [27-31] centers, were previously reported to have emission in 
the telecommunication bands that split under a magnetic field, and their ground states were believed to possibly have an unpaired electron spin. However, the most important photon-spin interface properties of these centers were unknown. Encouraged by this, and the knowledge that many defects in isotopically purified ${ }^{28} \mathrm{Si}$ boast exceptionally long electron [32,33] and nuclear [5] spin lifetimes, the goal of this work is to assess the $T$ center in ${ }^{28} \mathrm{Si}$ for its potential as a silicon telecom photon-spin interface.

Isotopically purified silicon improves upon the properties of photon-spin interfaces in multiple ways. For one, the coherence lifetimes of the spins are increased by removing the ${ }^{29} \mathrm{Si}$ nuclear spins as a source of dynamic magnetic noise [5]. Secondly, the inhomogeneous line broadening of optical transitions is reduced by removing static local band-gap and binding energy variations that arise from the silicon isotope mass mixture inherent in natural silicon [34]. Accordingly, to obtain the results in this work, unless otherwise specified, the sample under investigation is a piece of isotopically purified ${ }^{28} \mathrm{Si}$ crystal obtained from the Avogadro project [35] that is enriched to $99.995 \%{ }^{28} \mathrm{Si}$, with less than $10^{14} \mathrm{O} / \mathrm{cm}^{3}$ and less than $5 \times 10^{14} \mathrm{C} / \mathrm{cm}^{3}$. An ensemble of $T$ centers is fabricated within this sample by $10 \mathrm{MeV}$ electron irradiation followed by an annealing recipe in stages up to $450^{\circ} \mathrm{C}$ as described in Appendix A.

Here we report upon a variety of isotopic, luminescence, magneto-optical, thermal, bound exciton excited state, and spin properties of the $T$ center that further support its position as a photon-spin frontrunner in the technological race for a global quantum platform.

This paper is organized as follows. In Sec. II we begin with a brief introduction and review of what has been reported in the literature about the $T$ center. In Sec. III we present photoluminescence (PL) results, previously unobserved isotopic shifts, a Debye-Waller factor of 0.23(1) (equivalently written $0.23 \pm 0.01$ ) and a $0.94(1) \mu$ s emitter lifetime. In Sec. IV we apply photoluminescence excitation (PLE) spectroscopy to reveal an ensemble ZPL full-width half-maximum linewidth as narrow as 33(2) $\mathrm{MHz}$, and report upon the temperature dependence of the ZPL, whose linewidth, position, and amplitude all vary with temperature over the range $1.2-4.2 \mathrm{~K}$ in a predictable manner. In Sec. V we use PLE to identify the energies of many of the $T$ center's bound exciton (BE) excited states. In Secs. VI-VIII we study $T$ centers under an applied magnetic field. In Sec. VI we show magnetophotoluminescence, which reveals that the $T$ center has a number of different orientations that become inequivalent when a magnetic field is applied in an arbitrary direction. In Sec. VII we reveal a hyperfine interaction with the $T$ center's hydrogen nuclear spin, and explore the combination of continuous-wave spin resonance and resonant optical excitation for electron and nuclear spin state hyperpolarization and readout. In Sec. VIII we study a specific orientational subset to measure spin qubit characteristics using pulsed magnetic resonance, and demonstrate Hahn-echo $T_{2}$ lifetimes beyond $1 \mathrm{~s}$. We conclude with a discussion of prospects and future work. The appendices include technical details on the sample preparation methods and the experimental methods used in this work.

\section{REVIEW}

Luminescent silicon defects have been studied for decades, with the vast majority of this work taking place before the advent of quantum technology hardware development in the late 1990s. In the 1970s, luminescent defects in silicon, and in particular sharp spectral features created as a result of irradiation and/or heat treatment, were often labeled with a letter. A number of these so-called radiation damage centers have been studied extensively $[22,36]$. These centers can be formed by electron, neutron, or ion radiation damage followed by an annealing treatment, although some radiation damage centers, including the $T$ center, can be created with heat treatment alone $[20,25]$. Most centers in this category have ZPL transitions that are unsplit by magnetic fields, with no unpaired electron spins in their unexcited states, limiting their usefulness as photon-spin interfaces. The $T$ [18-31], $I$ [24-27], and $M$ [27-31] centers are notable exceptions to this general trend. The little-studied $T$ center was thought to have a spin $-\frac{1}{2}$ to spin $-\frac{1}{2}$ optical transition directly in the telecommunications $O$-band, although the composition of the ground state was in dispute $[19,20]$.

Level structure.--In 1981, Minaev and Mudryi [18] discovered that the ZPL of the " $T$ line" photoluminescence feature near $935 \mathrm{meV}$ was in fact a doublet. In 1985, Irion et al. [19] confirmed that the $1.8 \mathrm{meV}$-split doublet (later estimated in Ref. [20] to be $1.75 \mathrm{meV}$ ) is a result of two states in the same defect, which we refer to as $\mathrm{TX}_{0}$ and $\mathrm{TX}_{1}$. In the same work, Irion et al. presented the nonlinear and orientation-dependent stress dependence of the $T$ center's $\mathrm{TX}_{0}$ and $\mathrm{TX}_{1} \mathrm{ZPL}$ optical transitions, revealing a rhombic- $I\left(C_{2 v}\right)$ defect symmetry. Later studies [20] revealed even more splitting under stress, indicating that the $T$ center is of monoclinic $I\left(C_{1 h}\right)$ symmetry.

Based upon early magnetic field dependence studies [19], Irion et al. concluded that the $\mathrm{TX}_{0} \mathrm{ZPL}$ consists of a transition between a level possessing a highly anisotropic spin $-\frac{1}{2}$ particle and a level possessing a highly isotropic spin $-\frac{1}{2}$ particle. The isotropic and anisotropic spin $g$ factors were determined in Ref. [19]. Irion et al. proposed, erroneously, that an anisotropic hole spin and an isotropic electron spin both reside in the bound exciton state, which this work disproves. The magnetic resonance results in Sec. VII are consistent with the later model proposed by Safonov et al. [20]. In this model the $T$ ground state has an unpaired electron, and the TX state includes an additional bound exciton. The two bound electrons in the TX 
state pair into a spin-0 singlet state and the unpaired spin$3 / 2$ hole spin state is split by the reduced symmetry of the defect, which can be modeled as an internal stress, into two doublets, $\mathrm{TX}_{0}$ and $\mathrm{TX}_{1}$. The unpaired hole spin determines the magnetic splitting of the $\mathrm{TX}_{0}$ state.

The total binding energy of the electron-hole pair is approximately $235 \mathrm{meV}$, whereas the bound exciton is known to thermally disassociate around $40 \mathrm{~K}$ [19], corresponding to a binding energy of approximately $22 \mathrm{meV}$ [19] or approximately $32 \mathrm{meV}$ [20], which reflects the binding energy of the hole to the negatively charged $T^{-}$ center. A first look at ZPL shifts and temperature shifts of radiation damage centers in isotopically purified ${ }^{30} \mathrm{Si}$ was undertaken in Ref. [23]. A $T$ center level diagram, which summarizes these earlier findings, is shown in Fig. 1.

Atomic composition and formation.-Studies in 1985 [19], and then later in 1996 [20], identified the presence of at least one carbon atom and one hydrogen atom in the $T$ center, by observing shifts and splittings of the $T$ center $\mathrm{TX}_{0} \mathrm{ZPL}$ with the incorporation of ${ }^{13} \mathrm{C}$ and deuterium in the studied samples. Furthermore, a fourfold splitting observed in a local vibrational mode (LVM) seen in PL revealed that there are two inequivalent carbon atoms
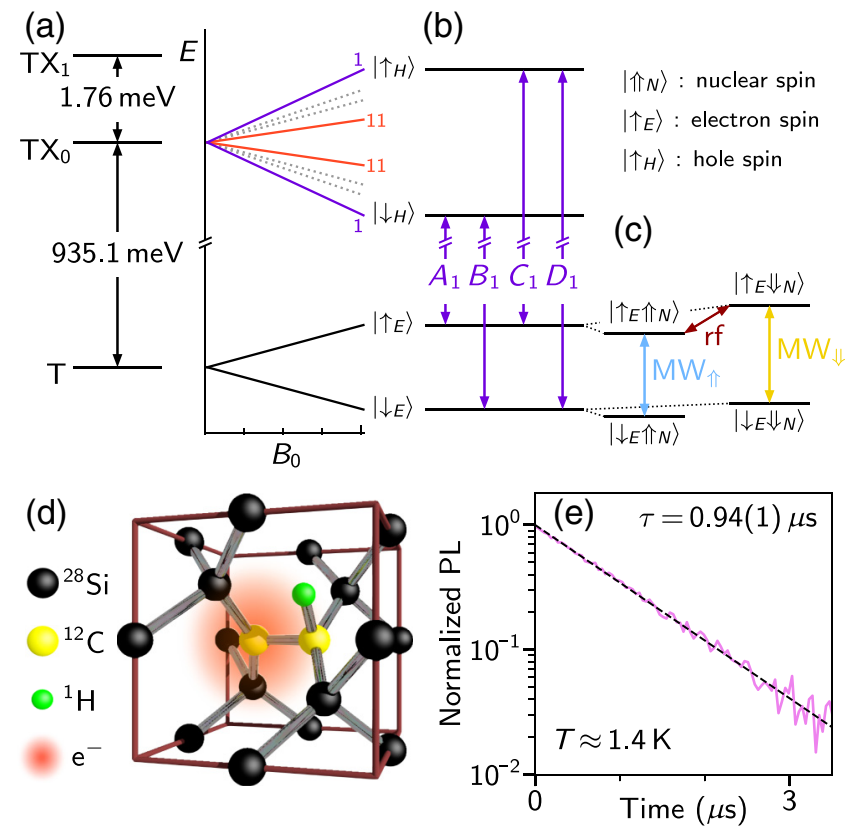

FIG. 1. (a) Level structure and magnetic field dependence of the ground $T$ level and the bound exciton $\mathrm{TX}_{0}$ state. The anisotropic unpaired hole spin in $\mathrm{TX}_{0}$ reveals 11 distinct orientational subsets. (b) The optical transitions $A_{1}, B_{1}, C_{1}, D_{1}$ correspond to those available to a single orientational subset, here subset 1. (c) Schematic of the spin levels and transitions of subset 1, relevant to the discussion in Secs. VI-VIII, under our experimental conditions. The nuclear spin states are not optically resolvable. (d) Atomic structure of the $T$ center as proposed in Ref. [20]. (e) Lifetime measurements of the $\mathrm{TX}_{0} \mathrm{BE}$. present in the defect [20]. There was early evidence in 1981 that oxygen is not involved in the formation of the $T$ center (as opposed to the $I$ center) [18]. The resemblance of the $T$ center with Al1 [37] and Ga1 [38] defects, with regard to LVM shifts, carbon isotope shift, and alleged symmetry, led to early speculation that boron was involved in the atomic structure of the $T$ center $[19,38]$ - there is however no direct evidence supporting this conjecture.

$A b$-initio cluster calculations [20,21], drawing upon a detailed analysis of the PL sideband LVM data first presented in Ref. [19] and expanded upon in Ref. [20], predicted an atomic structure for the $T$ center. In this model, two carbon atoms are directly bonded and together share the substitutional site of a silicon atom; one of these carbon atoms is terminated with a hydrogen atom, leaving an unpaired electron dangling bond on the other carbon atom [20]. This model is visualized in Fig. 1(d). The paramagnetic nature of the $T, I$, and $M$ centers in their neutral ground states are consistent with the presence of one hydrogen atom in each of these defects [27].

T center formation. - While it has been suggested that $\mathrm{M}$ centers are involved in the formation of $T$ centers [19,27], it has been claimed [25] that $M$ centers have never been observed in nonirradiated material, as opposed to $T$ centers. From ab-initio studies [20,21], the suggested $T$ center formation mechanism is the capture of an interstitial $C$ $H$ pair by a substitutional carbon atom [20-22]. $T$ centers have been observed both in Czochralski grown and float zone grown silicon [20], and the major step common to all studies is the need to apply a heat treatment with temperature between $350^{\circ} \mathrm{C}$ and $600^{\circ} \mathrm{C}$. Hydrogen can be already present in the silicon, or introduced by water vapour or gaseous hydrogen during the thermal anneal [25]. It has been observed that excess hydrogen in the silicon sample passivates the $T$ center [21], rendering it optically inactive.

\section{LUMINESCENCE SPECTRA}

In the PL experiments using nonresonant excitation in this work, whose technical details are given in Appendix B, above-band-gap light creates free carriers that pair up to form free excitons, which in turn are captured by $T$ centers, as well as other defects in the sample. Once bound to a $T$ center, the bound exciton recombines and, with some probability (given by the quantum radiative efficiency), this process generates a photon. Of these radiative cases, with some probability (given by the Debye-Waller factor [39]), this process generates a photon in the ZPL without any accompanying vibrational excitations (phonons or LVM), and the remainder of the time it produces vibrational excitations as well as light in the phonon sideband.

The lifetime of the TX level can be measured by applying pulsed above-band-gap excitation and measuring the luminescence decay of light emitted into the ZPL line, as described in Appendix B. This data is shown in Fig. 1(e) 
and reveals a $\mathrm{TX}_{0} \mathrm{BE}$ lifetime of 0.94(1) $\mu$ s. Repeating these lifetime measurements using a range of silicon samples with different defect concentrations at either 1.4 or $4.2 \mathrm{~K}$ reveals the same lifetime values within the margin of error. This increases our confidence that this reported lifetime is inherent to the $T$ center and is not due to, for example, the free-exciton decay time. These excited state lifetimes are sufficiently long to consider the excitonic degree of freedom as an additional local quantum resource.

The radiative quantum efficiency of this optical transition is presently unknown. Silicon's maximum phonon energy of approximately $65 \mathrm{meV}$ implies that pure nonradiative decay would require the simultaneous emission of at least 15 phonons. We are unable to observe any sample conductivity changes due to nonradiative Auger recombination processes, which is a highly successful method for measuring the nonradiative BE decay of low-concentration phosphorus donors in silicon [5]. Working under the assumption that the recombination lifetime is entirely from radiative processes, this $\mathrm{BE}$ lifetime corresponds to a total transition dipole moment of 1.52(1) Debye and a ZPL transition dipole moment of 0.73(2) Debye. From singlecenter linewidth upper bounds of 33(2) $\mathrm{MHz}$ as presented in Sec. IV, the above ZPL transition dipole moment would give a single-defect cooperativity of 1 in a photonic cavity with a $Q$ factor of $1 \times 10^{4}$ with a realistic mode volume of $(\lambda / n)^{3}$.

In Fig. 2 we report PL spectra of $T$ centers in silicon. This spectrum agrees with previous $T$ center PL spectra in the literature [19]. A low-resolution view of the Tcenter ZPL and phonon sideband is shown in Fig. 2(a), which includes sharp features like the LVM replica labeled $L_{2}$ following Ref. [20]. In Figs. 2(b)-2(d) we show highresolution spectra around the $\mathrm{TX}_{0} \mathrm{ZPL}$. The ${ }^{\text {nat }} \mathrm{Si}$ spectrum shown in Fig. 2(b) is from the brightest $T$ center sample in our possession, which was electron irradiated and heat treated in 1995 and stored at room temperature since then. Additional sample details are presented in Appendix A.

In Fig. 2(a) the phonon sideband relative to the normalized ZPL is shown. Other luminescent defects in the sample may also contribute to the observed PL signal, as can clearly be seen from the broad PL at energies both higher and lower than the $\mathrm{TX}_{0} \mathrm{ZPL}$. In order to measure the Debye-Waller factor accurately, we suppress this non- $T$-related PL by employing resonant PL spectroscopy, as described in Appendix B. The resulting resonant PL phonon sideband spectra, taken with the same resolution as the matching above-band-gap PL data, and with the silicon laser Raman line subtracted, is shown in Fig. 1(a). The sharp LVM feature $L_{2}$ is used to normalize the two PL traces so that an accurate Debye-Waller factor of 0.23(1) can be determined.

In Fig. 2(b) we report the first high-resolution optical spectroscopy of the $\mathrm{TX}_{0} \mathrm{ZPL}$ of $T$ centers in ${ }^{28} \mathrm{Si}$. As has been observed with group-V donors [34,40], group-VI

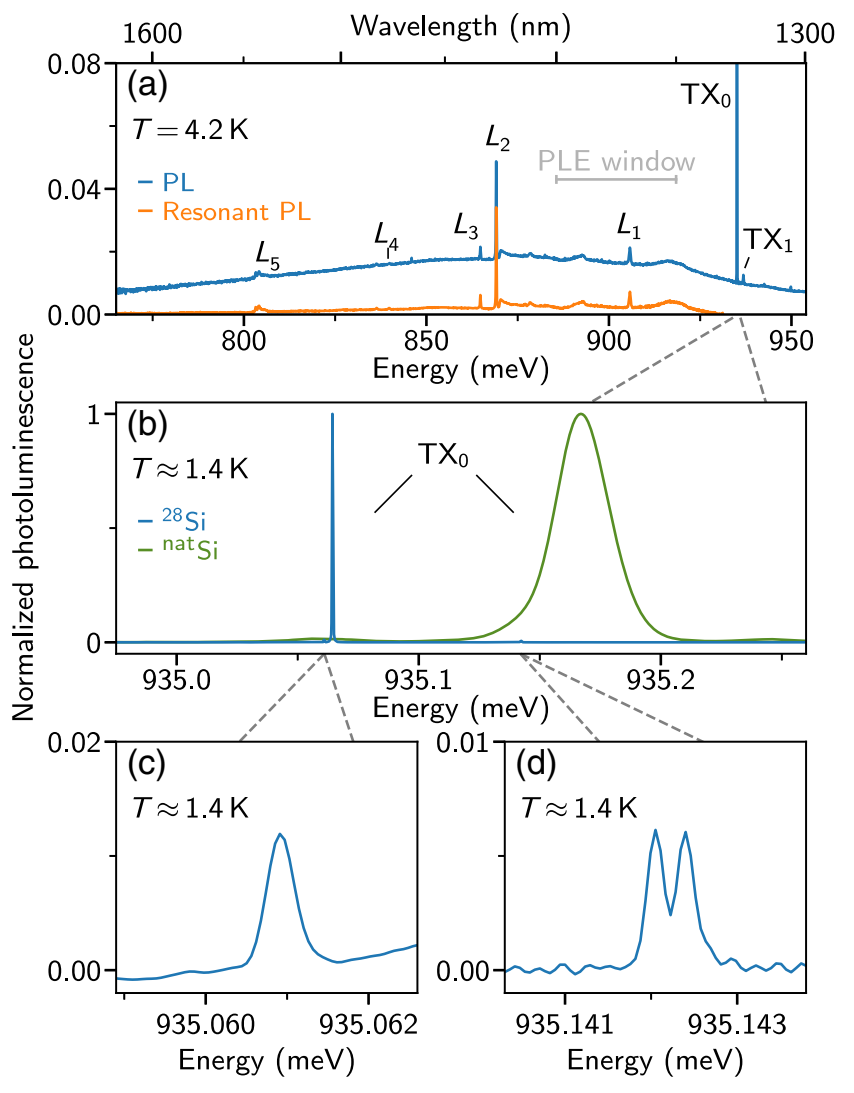

FIG. 2. (a) PL spectra of the $T$ center using above-gap and resonant excitation. The above-gap PL is normalized to a $T$ line $\left(\mathrm{TX}_{0}\right)$ intensity of unity, and the resonant PL is normalized to the above-gap PL using the $L_{2}$ local vibrational mode intensity. Together this data is used to extract a Debye-Waller factor of 0.23(1). The detection window used for the PLE experiments in Secs. IV, VII, and VIII is shown in grey. (b) High-resolution above-gap PL spectra of the $T$ center $\mathrm{TX}_{0} \mathrm{ZPL}$ in ${ }^{\text {nat }} \mathrm{Si}$ and in enriched ${ }^{28} \mathrm{Si}$. Weak features in the ${ }^{28} \mathrm{Si}$ spectrum attributed to ${ }^{13} \mathrm{C}$ isotope-shifted replicas for the $T$ center's two inequivalent carbon atoms are visible at (c) 935.060 93(7) $\mathrm{meV}$, with an integrated area ratio of $1.1(1) \%$ relative to the $\mathrm{TX}_{0} \mathrm{ZPL}$, and (d) a doublet at $935.14204(10) \mathrm{meV}$ and $935.14240(6) \mathrm{meV}$, with a combined integrated ratio of $1.16(9) \%$ relative to the $\mathrm{TX}_{0} \mathrm{ZPL}$.

double donors [6,7], and other radiation damage centers [12], the removal of inhomogeneous local isotopic variations in the silicon lattice has a significant effect on the observed linewidth of the $T$ center ZPL in isotopically enriched ${ }^{28} \mathrm{Si}$. In high-purity ${ }^{\text {nat }} \mathrm{Si}$ we observe a linewidth of 26.9(8) $\mu \mathrm{eV}$, whereas in ${ }^{28} \mathrm{Si}$ the linewidth is below the $0.25 \mu \mathrm{eV}$ lower-resolution limit of our Fourier transform infrared spectrometer. A higher-resolution temperaturedependent linewidth study using PLE techniques presented in Sec. IV reveals ${ }^{28} \mathrm{Si}$ ensemble ZPL linewidths to be as low as $0.14(1) \mu \mathrm{eV}(33(2) \mathrm{MHz}$ ) - nearly a 200-fold reduction in linewidth. In addition to a narrowing of the $\mathrm{TX}_{0}$ line, we also observe a peak wavelength shift from $935.167(2) \mathrm{meV}$ in ${ }^{\text {nat }} \mathrm{Si}$ to $935.0643(1) \mathrm{meV}$ in ${ }^{28} \mathrm{Si}$ at 
1.4 K. Similar spectral shifts, due to the dependence of the band gap on average isotopic mass, electron-phonon coupling, and the defect binding energy, have been widely documented in silicon [34], including in work studying $T$ centers in ${ }^{30} \mathrm{Si}$ [23].

A closer look at the $1.4 \mathrm{~K}$ high-resolution ${ }^{28} \mathrm{Si} \mathrm{PL}$ spectrum in Fig. 2(b) reveals the presence of a negatively shifted satellite at $935.06093(7) \mathrm{meV}$ [Fig. 2(c)] and a positively shifted doublet at $935.14204(10) \mathrm{meV}$ and 935.142 40(6) meV [Fig. 2(d)] with integrated area ratios relative to the main ZPL of $1.1(1) \%$ and $1.16(9) \%$, which are consistent with the $1.1 \%$ natural isotopic abundance of ${ }^{13} \mathrm{C}$ and the fact that the $T$ center contains two inequivalent carbon atoms. Both features provide new understanding afforded by the isotopically purified ${ }^{28} \mathrm{Si}$ host material. The negatively shifted ${ }^{13} \mathrm{C}$ peak is fully obscured in ${ }^{\text {nat }} \mathrm{Si}$. The positively shifted feature, although characterized at low resolution in earlier isotopic composition studies [19,20], reveals a previously hidden structure: an $86(20) \mathrm{MHz}$ splitting that is unobservable in ${ }^{\text {nat }} \mathrm{Si}$ and is possibly connected to the ${ }^{13} \mathrm{C}$ nuclear spin.

\section{ZPL TEMPERATURE DEPENDENCE}

PLE experiments with tunable single-frequency lasers, as described in Appendix B, can measure linewidths below the resolution limits of high-resolution spectrometers used for PL spectroscopy. Here we scan a laser over the $\mathrm{TX}_{0}$ ZPL at different sample temperatures and detect sideband photons with wavelengths within the PLE detection window shown in Fig. 2(a). The raw data as well as the fit results are shown in Fig. 3.

Lorentzian line shapes provide an excellent fit to the raw data across the entire temperature range studied, giving linewidths of $1.18(3) \mu \mathrm{eV}$ at $4.2 \mathrm{~K}$ and $0.14(1) \mu \mathrm{eV}$ at $1.4 \mathrm{~K}$. Fit parameters for all temperatures are plotted in Figs. 3(b)-3(d). The linewidth versus temperature data is fitted to a thermally induced transition model [12], i.e.

$$
\Gamma(T)=P_{0}+\frac{P_{T}}{\exp \left(E_{a} / k_{B} T\right)-1},
$$

yielding $P_{0}=0.137(8) \mu \mathrm{eV}, P_{T}=78(12) \mu \mathrm{eV}$, and an activation energy $E_{a}=1.6(1) \mathrm{meV}$. This is in reasonable agreement with the 1.76(1) $\mathrm{meV} \mathrm{TX}_{0}$ to $\mathrm{TX}_{1}$ state splitting measured in PL at $4.2 \mathrm{~K}$. This demonstrates that the thermal broadening of the $\mathrm{TX}_{0}$ line is due to thermally activated transitions between $\mathrm{TX}_{0}$ and $\mathrm{TX}_{1}$.

Evidence for the transition from a thermally broadened homogeneous line to an inhomogeneous line includes the PLE signal amplitude and integrated area decrease at temperatures below $2.4 \mathrm{~K}$, as seen in Fig. 3(c). A similar signal decrease is not observed in PL. In an inhomogeneously broadened line, local degrees of freedom such as spin states can shift the position of a given center's ZPL.
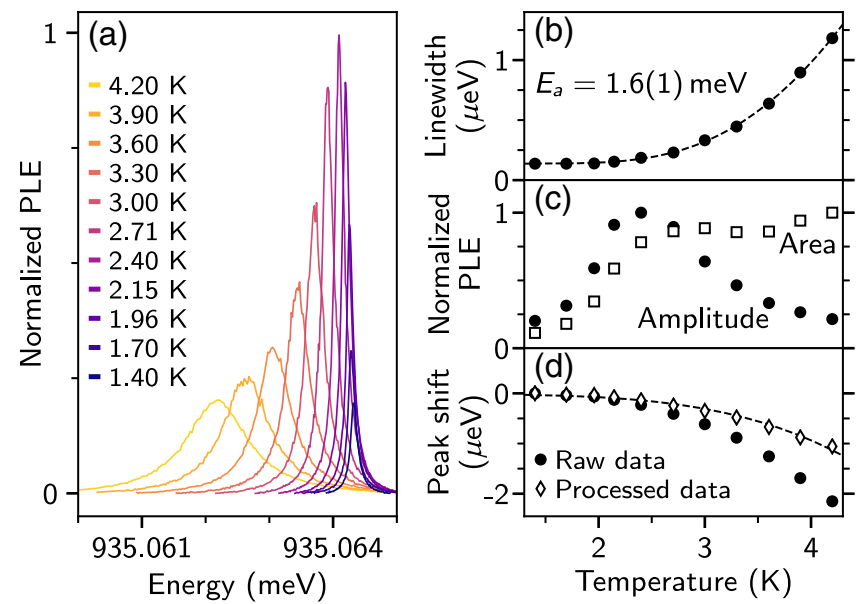

FIG. 3. (a) Photoluminescence excitation spectra of the $\mathrm{TX}_{0}$ ZPL transition as a function of temperature, normalized to the $2.4 \mathrm{~K}$ spectrum. The decrease in the PLE signal at low temperatures indicates a shift from homogeneous to inhomogeneous broadening, as discussed in the text. (b)-(d) Lorentzian fit parameters for the spectra in panel (a). Error bars are smaller than the size of the data points. (b) The ZPL linewidth increases as a function of temperature following a thermally induced transition model with an activation energy of 1.6(1) $\mathrm{meV}$ (details in the text). (c) Normalized ZPL amplitude (filled circles) and integrated area (open squares) as a function of temperature. (d) Peak position shift, where the filled circles show the raw data and the open diamonds show the shift when corrected for hydrostatic pressure effects.

If resonant optical driving changes these degrees of freedom, for example by flipping a spin state, that center's cycling transition frequency may no longer be in resonance with the optical driving frequency and the number of emitted photons will drop accordingly. This effect increases as the ratio of homogeneous to inhomogeneous linewidth decreases with lower temperatures. The lower bound on the homogeneous linewidth, from the temperatureindependent lifetime data, is $1 /(2 \pi \times 940 \mathrm{~ns})=0.169(2)$ $\mathrm{MHz}$ (or $0.700(8) \mathrm{neV}$ ). The observation that an inhomogeneously broadened line is Lorentzian is not uncommon and could be due to strain from residual impurities, random electric fields from ionized impurities, or crystal damage [41]. Further data in support of this interpretation are given in Sec. VII.

The peak position also changes with temperature, as seen in Fig. 3(d). The peak position shift includes both hydrostatic pressure and temperature shift effects, since the temperature is controlled by reducing the gas pressure over the liquid helium. As is the case with many silicon defects, the sample's ambient pressure should have a linear effect on the peak position because of the band-gap energy shift [42]. To measure the constant governing this linear shift, we compare two spectra-one in liquid ${ }^{4} \mathrm{He}$, the other in gas ${ }^{4} \mathrm{He}$ - and make sure that they are at the same temperature by comparing their low-power (unsaturated) 
linewidths, which assumes that pressure changes have a negligible effect on the ZPL linewidth. Using this technique, the resulting constant is $1.47(34) \times 10^{-9} \mathrm{eV} /$ Torr, which we subtract from the total peak position shift. The remaining peak shift is well fitted by an $A T^{4}$ temperature dependence for $A=-3.58 \mathrm{neV} / T^{4}$, which is proportional to the known $T^{4}$ behavior of the band-gap energy in the asymptotic low-temperature limit [42].

\section{BOUND EXCITON EXCITED STATES}

In the neutral ground state the $T$ center contains an unpaired electron. During the lifetime of the bound exciton the TX center can be thought of as a pseudo-acceptor. Characteristics of this pseudo-acceptor include the existence of effective-mass-like excited states for the hole not unlike those of group-III acceptors such as boron. These excited states are labeled $N \Gamma_{K}^{+}$or $N \Gamma_{K}^{-}$for evenor odd-parity states, respectively, counted with principal quantum numbers $N=1, \ldots$, where $K$ indicates the state's symmetry group (See Ref. [43] and the references therein).

The ground state of TX has a hole in an acceptor-like fourfold degenerate $1 \Gamma_{8}^{+}$state, split into the two Kramers doublets by the strain field of the defect, giving rise to $\mathrm{TX}_{0}$ and $\mathrm{TX}_{1}$. The electronic excited state spectrum of TX is thus expected to be acceptor-like, as seen before for other pseudo-acceptor isoelectronic bound excitons (IBEs) in silicon [44]. However, the excited state spectra of pseudo-acceptor IBEs as seen in PLE [44] differ from the well-known absorption spectra of shallow acceptors such as boron, since the IBE spectra involve the creation of an electron-hole pair, resulting in strong transitions to

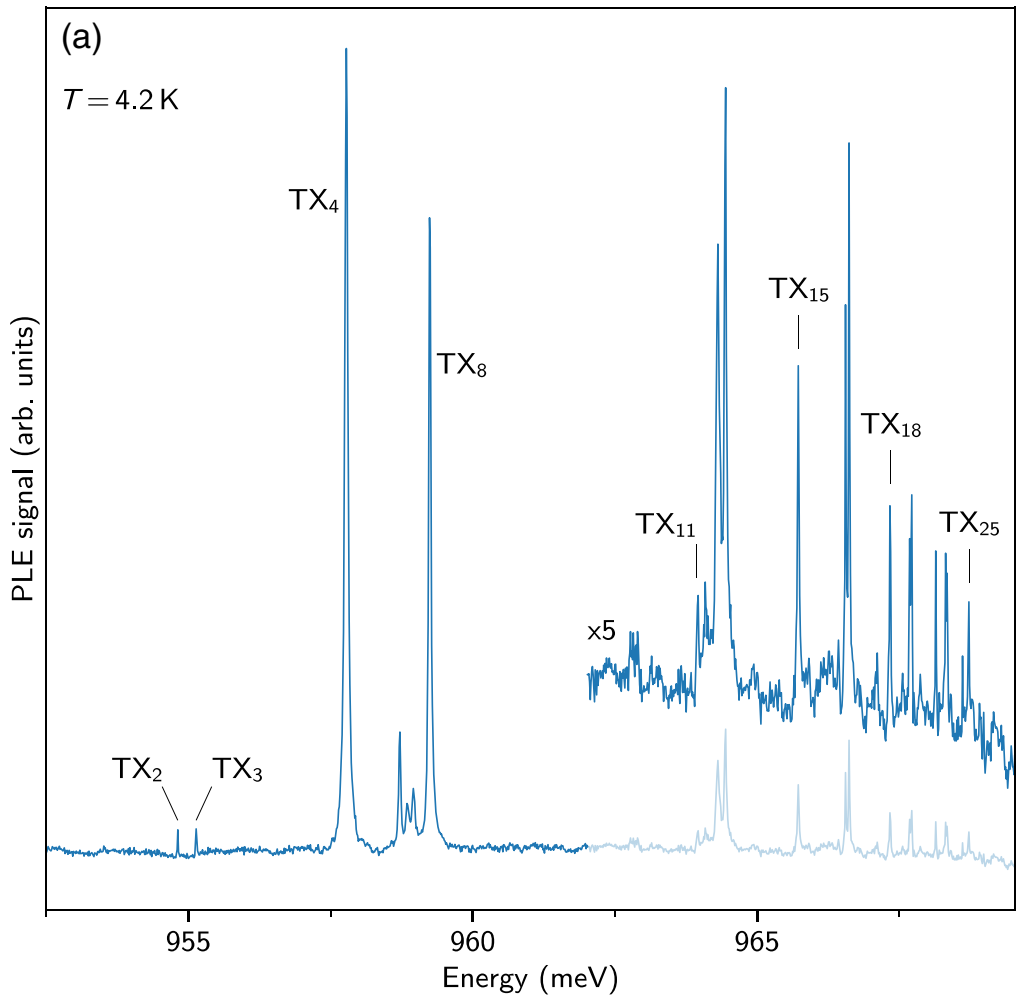

(b)

\begin{tabular}{lrrc}
\hline \hline Line & Energy $(\mathrm{meV})$ & Amplitude & State \\
\hline $\mathrm{TX}_{0}$ & 935.0622 & $(\mathrm{PL} 4.2 \mathrm{~K})$ & $1 \Gamma_{8}^{+}$ \\
$\mathrm{TX}_{1}$ & 936.82 & $(\mathrm{PL} 4.2 \mathrm{~K})$ & 11 \\
$\mathrm{TX}_{2}$ & 954.81 & 0.026 & $1 \Gamma_{8}^{-}$ \\
$\mathrm{TX}_{3}$ & 955.14 & 0.027 & $" 1$ \\
$\mathrm{TX}_{4}$ & 957.78 & 1.000 & $2 \Gamma_{8}^{+}$ \\
$\mathrm{TX}_{5}$ & 958.72 & 0.147 & $2 \Gamma_{8}^{-}$ \\
$\mathrm{TX}_{6}$ & 958.85 & 0.058 & $" 1$ \\
$\mathrm{TX}_{7}$ & 958.96 & 0.077 & $" 1$ \\
$\mathrm{TX}_{8}$ & 959.25 & 0.790 & $2 \Gamma_{8}^{+}$ \\
$\mathrm{TX}_{9}$ & 962.80 & 0.009 & $3 \Gamma_{8}^{-}$ \\
$\mathrm{TX}_{10}$ & 962.89 & 0.009 & $" 1$ \\
$\mathrm{TX}_{11}$ & 963.96 & 0.025 & $1 \Gamma_{6}^{-}$ \\
$\mathrm{TX}_{12}$ & 964.09 & 0.028 & $1 \Gamma_{7}^{-}$ \\
$\mathrm{TX}_{13}$ & 964.31 & 0.112 & $3 \Gamma_{8}^{+} \mathrm{b}$ \\
$\mathrm{TX}_{14}$ & 964.45 & 0.151 & 11 \\
$\mathrm{TX}_{15}$ & 965.73 & 0.082 & $1 \Gamma_{6}^{+}$ \\
$\mathrm{TX}_{16}$ & 966.56 & 0.097 & $4 \Gamma_{8}^{+}$ \\
$\mathrm{TX}_{17}$ & 966.62 & 0.137 & $" 1$ \\
$\mathrm{TX}_{18}$ & 967.34 & 0.047 & $2 \Gamma_{6}^{+}$ \\
$\mathrm{TX}_{19}$ & 967.68 & 0.039 & $5 \Gamma_{8}^{+}$ \\
$\mathrm{TX}_{20}$ & 967.72 & 0.050 & $" 1$ \\
$\mathrm{TX}_{21}$ & 968.14 & 0.036 & $3 \Gamma_{6}^{+}$ \\
$\mathrm{TX}_{22}$ & 968.32 & 0.035 & $6 \Gamma_{8}^{+}$ \\
$\mathrm{TX}_{23}$ & 968.34 & 0.030 & $" 1$ \\
$\mathrm{TX}_{24}$ & 968.61 & 0.010 & $4 \Gamma_{6}^{+}$ \\
$\mathrm{TX}_{25}$ & 968.73 & 0.023 & $7 \Gamma_{8}^{+}$ \\
\hline \hline & & &
\end{tabular}

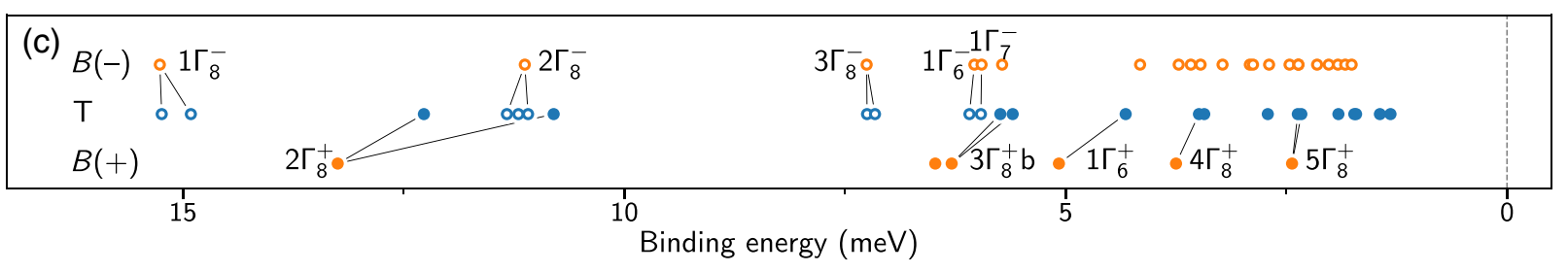

FIG. 4. (a) PLE spectrum revealing higher excited states of the $T$ center bound exciton level TX. (b) Transition energies of all the known TX states, where $\mathrm{TX}_{0}$ and $\mathrm{TX}_{1}$ are determined using PL, and the $\mathrm{TX}_{>1}$ are determined from the PLE data shown in (a). The PLE peak amplitudes are shown relative to that of the $\mathrm{TX}_{4}$ transition. The $N \Gamma_{8}^{+}$state labels for $N<7$ refer to the fourfold degenerate shallow acceptor states from which the two doubly degenerate TX states originate. This splitting can no longer be resolved for $7 \Gamma_{8}^{+}$ $\left(\mathrm{TX}_{25}\right)$. (c) The known binding energies of boron even-parity excited states $(B(+)$, filled circles) and odd-parity states $(B(-)$, open circles) are matched with the observed TX excited states shown in (a) in order to determine the hole binding energies of the TX states. The assignment of three TX transitions to $2 \Gamma_{8}^{-}$, while only two are expected, is discussed in the text. 
even-parity states beginning with the $1 \Gamma_{8}^{+}$ground state, while acceptor absorption transitions take an existing hole from the $1 \Gamma_{8}^{+}$acceptor ground state to predominantly odd-parity excited states [45].

The excited states of acceptors can be observed using a range of techniques [43]. In the case of the $T$ center's BE, a number of the excited states can be mapped out directly with PLE techniques. For the data presented in Fig. 4, a tunable single-frequency laser was scanned above the energy of the $\mathrm{TX}_{0,1}$ states, resonantly populating various TX excited states. We measured this excitation by detecting $\mathrm{TX}_{0} \mathrm{ZPL}$ luminescence, resulting from phonon cascade from the excited states down to the $\mathrm{TX}_{0}$ state, using a spectrometer and a single-photon detector as described in Appendix B. We report the higher excited states of the TX center in silicon.

The identification of the excited states given in Fig. 4(b) is obtained by matching the excited state spectrum of TX to the known even- and odd-parity excited state binding energies of boron in silicon [43,45], as shown in Fig. 4(c). Note that both the even- and odd-parity $N \Gamma_{8}$ states can be split into doublets by the strain field of TX, and this splitting decreases with increasing principal quantum number $N$. The observation of three components, $\mathrm{TX}_{5-7}$, where only two are expected for $2 \Gamma_{8}^{-}$, is not understood. It is possible that one of these components might result from the $1 \Gamma_{7}^{+}$ state [43] of the TX center. The even- and odd-parity $\Gamma_{6}$ and $\Gamma_{7}$ states are not split by strain. As expected, the chemical shifts, or central cell effects, are much smaller for the odd-parity states, which have a node at the binding center core, than for the even-parity states, where the central cell effects decrease rapidly with increasing $N$. Even-parity states above $5 \Gamma_{8}^{+}$are not known for boron, but we label TX states up to $7 \Gamma_{8}^{+}$based on the clear repetition of the $3 \Gamma_{8}^{+}-1 \Gamma_{6}^{+}-4 \Gamma_{8}^{+}$pattern.

By matching the $1 \Gamma_{6}^{-}, 1 \Gamma_{7}^{-}$, and $5 \Gamma_{8}^{+}$transitions of TX and boron, and the known binding energies $[43,45]$ of these states for boron, we arrive at the energy of $970.05(10) \mathrm{meV}$ required to promote a $T$ into a $T^{-}$plus a free hole with zero kinetic energy, or a hole binding energy for TX of 35.0(1) $\mathrm{meV}$. This agrees reasonably well with the earlier estimate of $32 \mathrm{meV}$ deduced from the thermal deactivation of the TX PL [20]. We note that these results provide the most complete existing matching of pseudo-acceptor IBE states with shallow acceptor states.

\section{MAGNETOPHOTOLUMINESCENCE}

The substantial reduction in optical inhomogeneous broadening afforded by the ${ }^{28} \mathrm{Si}$ host material encouraged the re-examination of the PL of $T$ centers under an applied

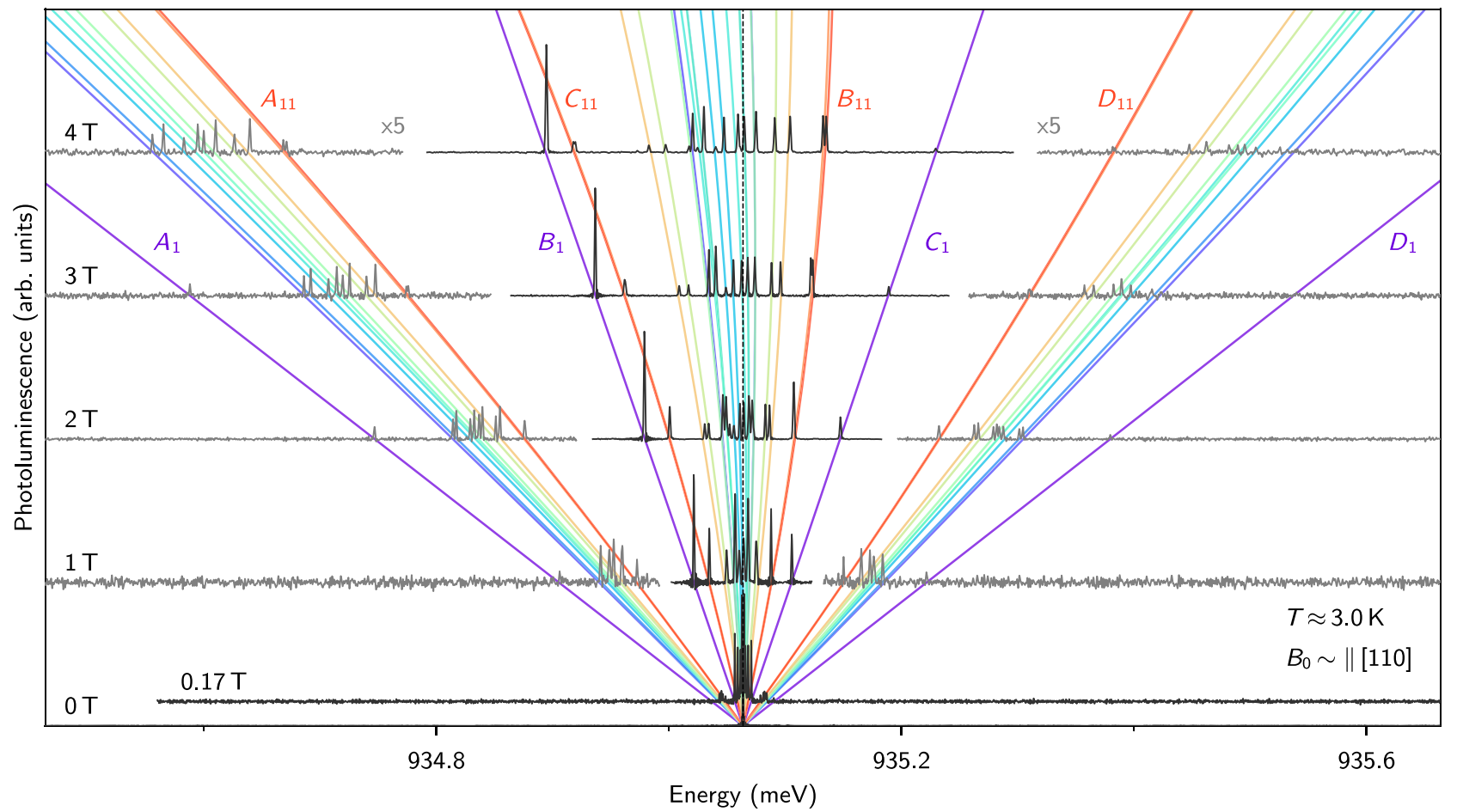

FIG. 5. Photoluminescence of the $T$ center TX $\mathrm{T}_{0}$ ZPL under an applied magnetic field with $B_{0}$ approximately parallel to the [110] crystal axis. The four optical transitions available to each identifiable orientational subset are grouped by color. Orientational subset 1 (purple) has optical transitions labeled $A_{1}, B_{1}, C_{1}, D_{1}$ in accordance with Fig. 1(b). Also labeled are the transitions of the subset having the smallest hole $g$ factor, $A_{11}, B_{11}, C_{11}$, and $D_{11}$. The colored lines are fits to Eq. (2), with resulting fit parameters given in Table I. A multiplier of $\times 5$ has been applied to the wings of the spectra to enhance the visibility of lower amplitude lines. 
magnetic field. As seen in Fig. 5, the PL spectrum is substantially richer in ${ }^{28} \mathrm{Si}$ than those of previous reported spectra in ${ }^{\text {nat }} \mathrm{Si}$ [19], even in the moderate magnetic fields explored here.

From the known $C_{1 h}$ symmetry of the center, we expect twelve possible orientational subsets of $T$ centers relative to an arbitrary $B_{0}$ field axis [46]. The unpaired spin $-\frac{1}{2}$ electron was known to be highly isotropic in contrast with the highly anisotropic spin $-\frac{1}{2}$ hole. Together this implies up to 48 observable optical transitions for a low-symmetry field axis, where each $T$ center orientational subset gives rise to four allowed optical transitions, as schematically shown in Fig. 1(b). Further optical substructure, for example due to nuclear spin Zeeman and hyperfine effects, could not be resolved directly in these ensemble measurements.

In Fig. 5 we reveal eleven $T$ center orientational subsets that are identified by color. Here we define "orientational subset 1" as an orientational subset addressable with optical signals $A_{1}, B_{1}, C_{1}, D_{1}$, which underpins the spin resonance studies in Secs. VII and VIII. Furthermore, in Sec. VII we reveal through magnetic resonance techniques that the purple subset with assigned $A_{1}, B_{1}, C_{1}, D_{1}$ labels in Fig. 5 addresses two near-degenerate orientational subsets, bringing the total observed number of orientational subsets in this work to twelve. Note that subset 1 has the largest hole $g$ factor of all the subsets. We also label the transitions of the subset having the smallest hole $g$ factor as $A_{11}, B_{11}$, $C_{11}$, and $D_{11}$. Note that the $A B C D$ labels indicate transitions connecting specific spin states, and not the energetic ordering of the transitions, so the energy of $B$ versus $C$ is reversed for subsets 4 through 11 .

Each orientational subset's optical transition frequencies are well fit to a spin Hamiltonian model with Zeeman terms for each of the unpaired ground state electron and excited state hole spins as well as a diamagnetic term. Specifically, we fit using the following set of four equations:

$$
\{A, B, C, D\}_{i}= \pm \frac{g_{E, i} \mu_{B}\left|\mathbf{B}_{0}\right|}{2 e} \pm \frac{g_{H, i} \mu_{B}\left|\mathbf{B}_{0}\right|}{2 e}+\chi_{i}\left|\mathbf{B}_{0}\right|^{2}
$$

Here $\{A, B, C, D\}_{i}$ are the peak position shifts from the zero-field value for subset $i, g_{E, i}$ is the ground state electron $g$ factor constant for orientational subset $i$ under our experimental conditions, $\mu_{B}$ is the Bohr magneton, $\mathbf{B}_{0}$ is the magnetic field vector, $e$ is the elementary charge, $g_{H, i}$ is the bound exciton hole $g$ factor for orientational subset $i$ under our experimental conditions, and $\chi_{i}$ is the diamagnetic shift for orientational subset $i$ under our experimental conditions. The $g_{E, i}$ are equal within error, with an average of 2.005(8). Results of the fit for the hole $g$ factors and diamagnetic shifts of all identifiable subsets are given in Table I.

The relative amplitudes of the four lines associated with a given orientational subset measured using PL are affected
TABLE I. TX $\mathrm{TX}_{0}$ hole spin $g$ factors and diamagnetic shift constants for the different $T$ center orientational subsets observed with this magnetic field axis (approximately parallel to the [110] crystal axis), whose colors and labels match the subsets labeled in Fig. 5.

\begin{tabular}{lcc}
\hline \hline$i$ & $\begin{array}{c}\mathrm{TX}_{0} \text { hole spin } g \text { factor } \\
g_{H, i}\end{array}$ & $\begin{array}{c}\text { Diamagnetic shift } \\
\chi_{i}\left(\mu \mathrm{eV} / T^{2}\right)\end{array}$ \\
\hline $1 \square$ & $-0.11(2)$ \\
2 & $3.457(7)$ & $-1.05(6)$ \\
3 & $2.233(9)$ & $-1.03(6)$ \\
4 & $2.165(14)$ & $-1.26(4)$ \\
5 & $1.970(12)$ & $-1.36(9)$ \\
6 & $1.871(22)$ & $-1.03(6)$ \\
7 & $1.851(14)$ & $-1.10(7)$ \\
8 & $1.770(8)$ & $-1.23(5)$ \\
9 & $1.596(6)$ & $-1.26(9)$ \\
10 & $1.497(11)$ & $-2.35(3)$ \\
11 & $1.082(7)$ & $-2.35(3)$ \\
\hline \hline
\end{tabular}

by: (i) spin selection rules, and (ii) the hole spin thermal populations, where we observe that the hole spin relaxation time is comparable to or shorter than the TX BE lifetime of $940 \mathrm{~ns}$. As discussed in the next section, the relative line intensities in spectra obtained by PLE in a magnetic field are quite different from those obtained using PL.

\section{CONTINUOUS-WAVE SPIN RESONANCE}

The many optical transitions seen in magnetophotoluminescence are not immediately observable under the resonant optical driving of a PLE measurement. As shown in the bottom trace of Fig. 6(a), without the application of any external signals, only a small central peak is visible under an applied magnetic field of $80 \mathrm{mT}$ when using the optically detected magnetic resonance apparatus described in Appendix B. The absence of most optical transitions in PLE is an expected consequence of ground state electron spin hyperpolarization resulting from spin-selective resonant excitation, as shown schematically in Fig. 6(b). Under such a model, the central peak remains visible because, for a few orientational subsets, the ground and exciton spin states share approximately the same $g$ factor value, which is to say that their $B$ and $C$ transitions almost overlap, and so both electron ground spin states can be continually optically excited at that central frequency and electron spin hyperpolarization does not occur.

To regain some of the structure observed in magnetophotoluminescence, resonant continuous-wave (CW) electron paramagnetic resonance (EPR) in the form of microwave (MW) irradiation can be applied to continually depolarize or mix the electron spin, as shown schematically in Fig. 6(c). Spin mixing depopulates the spin shelving state(s) that is (are) not being optically pumped and allows the optical excitation cycle to resume. 


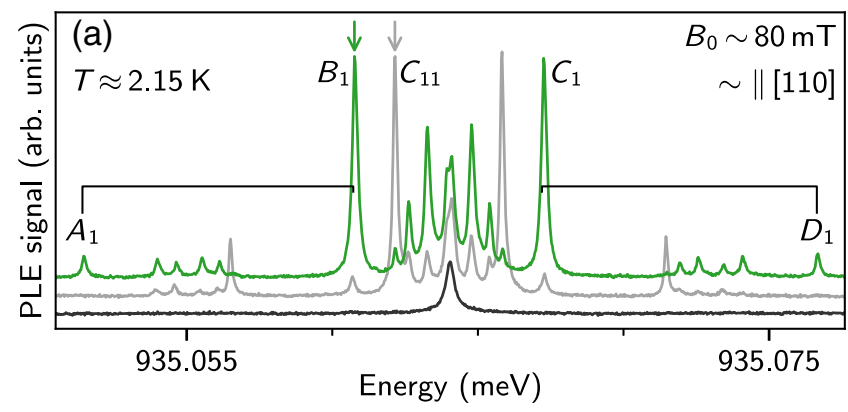

(b)

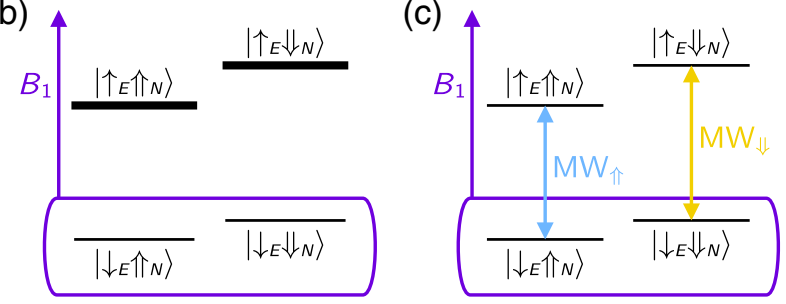

FIG. 6. (a) PLE spectrum of the $\mathrm{TX}_{0} \mathrm{ZPL}$ under a static magnetic field of about $80 \mathrm{mT}$ oriented approximately parallel to the [110] crystal axis, with and without electron spin depolarization. Line labels $A, B, C, D$ match those of Figs. 1(b) and 5. Brackets display the $g_{E}=2.005(8)$ electron splitting at this field. The black trace (bottom) is collected without any MW excitation. The green trace (top) is obtained when pumping $\mathrm{MW}_{\Uparrow}=2.2504$ $\mathrm{GHz}$ and $\mathrm{MW}_{\Downarrow}=2.2533 \mathrm{GHz}$, which maximizes the signal from subset 1 . The grey trace (middle) includes a single central MW signal that drives both $\mathrm{MW}_{\Uparrow}$ and $\mathrm{MW}_{\Downarrow}$ from subset 11. (b),(c) Ground state energy levels created by the hyperfine interaction between the electron spin and the nuclear spin, as per Eq. (3). The schematic level diagrams picture electron spin hyperpolarization in (b) and electron spin mixing in (c). Shelving is indicated by a thicker energy level.

Overall the spin Hamiltonian for the $T$ ground state $\mathcal{H}_{\mathcal{T}}$ with two ${ }^{12} \mathrm{C}$ constituents is given by

$$
\mathcal{H}_{\mathcal{T}}=\mu_{B} \mathbf{B}_{0} \mathbf{g}_{\mathbf{E}} \mathbf{S}+\mu_{N} g_{N} \mathbf{B}_{0} \mathbf{I}+\mathbf{h S A I}
$$

where $\mu_{B}$ is the Bohr magneton, $\mathbf{B}_{0}$ is the magnetic field vector, $\mathbf{g}_{\mathbf{E}}$ is the electron spin $g$ factor tensor that is approximately isotropic with $g_{E}=2.005(8), \mathrm{S}$ is the electron spin vector, $\mu_{N}$ is the nuclear spin magneton, $g_{N}$ is the hydrogen nuclear spin $g$ factor, $\mathbf{I}$ is the hydrogen nuclear spin vector, $h$ is the Planck constant, and $\mathbf{A}$ is the hyperfine tensor. To obtain the results in this work, we find $\mathbf{A}$ is approximated well by a constant $A_{\text {eff }}$ for orientational subsets 1 and 11 and field vector $B_{0}$ approximately parallel to the [110] crystal axis. This spin Hamiltonian gives rise to the level structure shown in Figs. 6(b) and 6(c) under our experimental conditions, where we label the two conditional $\mathrm{MW}$ frequencies $\mathrm{MW}_{\Uparrow}$ and $\mathrm{MW}_{\Downarrow}$ according to their respective nuclear spin states.

Even though we will find that these orientational subsets have small hyperfine splittings due to the nuclear spin of the hydrogen, a relatively strong MW field resonant with the $\mathrm{g}_{E}=2.005(8)$ transition frequency at approximately $80 \mathrm{mT}$ is sufficient to simultaneously pump both $\mathrm{MW}_{\Uparrow}$ and $\mathrm{MW}_{\Downarrow}$ resonances thanks to power broadening, partially depolarizing the ground state electron spins and strengthening the central PLE component, as well as allowing many of the other PLE components to be observed, as shown in the middle trace (grey data) of Fig. 6(a). From this observation we can definitively conclude that the isotropic unpaired electron spin occurs in the unexcited $T$ center, and not in the TX BE state.

We find that, for specific orientational subsets, specific pairs of microwave frequencies centered on the $g_{E}=$ $2.005(8)$ value mix the ground state spins most effectively, maximizing the PLE signal from that orientational subset. For example, the top trace of Fig. 6(a), where the subset-1 signal is maximized with $\mathrm{MW}_{\Uparrow}=2.2504 \mathrm{GHz}$ and $\mathrm{MW}_{\Downarrow}=2.2533 \mathrm{GHz}$, reveals an effective hyperfine splitting $\left|A_{\text {eff }}\right|=2.9 \mathrm{MHz}$ for subset 1 (we will later show that closer study of subset 1 actually reveals two different values of $\left.\left|A_{\text {eff }}\right|\right)$. The differences between the top and middle traces of Fig. 6(a) demonstrate that the hydrogen spin hyperfine interaction of the $T$ center is anisotropic.

The specific MW frequency combination used to generate the green data in Fig. 6(a) is chosen as a result of magnetic resonance spectroscopy experiments. This optimization process is described next.

For orientational subset $i$, the tunable laser is first set to a $B_{i}$ or $C_{i}$ transition energy as determined in Sec. VI. For example, for orientational subset 1 , this energy is indicated by the letter $B_{1}$ (green arrow) in Fig. 6(a). In this case both the $B_{1}$ and $D_{1}$ optical transitions promote the $T\left|\downarrow_{E}\right\rangle$ electron states to $\mathrm{TX}_{0}$, consequently hyperpolarizing the electron into the $\left|\uparrow_{E}\right\rangle$ electron state; the $A_{1}$ and $C_{1}$ optical transitions generate the opposite electron spin hyperpolarization. Two tunable MW sources are initially each set to the central MW frequency given by the electron spin $g$ factor value. Following this, a symmetric MW frequency sweep is applied to reveal the pair of MW frequencies, split by the hydrogen hyperfine interaction, able to most effectively depolarize that particular orientational subset and generate an optically detected magnetic resonance (ODMR) signal.

For a single orientational subset and accurately centered initial MW frequencies, the result of this sweep should display a single peak at the effective hyperfine value associated with that orientational subset at the chosen magnetic field direction. The result of this scan applied to orientational subset 11 , with the laser set to the $\mathrm{C}_{11}$ optical transition [grey arrow in Fig. 6(a)], reveals that the subset-11 PLE signal is maximized for a MW frequency difference of $\left|A_{\text {eff }}\right|=0.40$ (3) MHz. The result of this scan applied to orientational subset 1 is shown in Fig. 7. The doublet observed in Fig. 7 reveals that orientational subset 1 actually comprises a pair of orientational subsets, hereafter referred to as subset 1 and subset $1^{\prime}$, thus bringing 


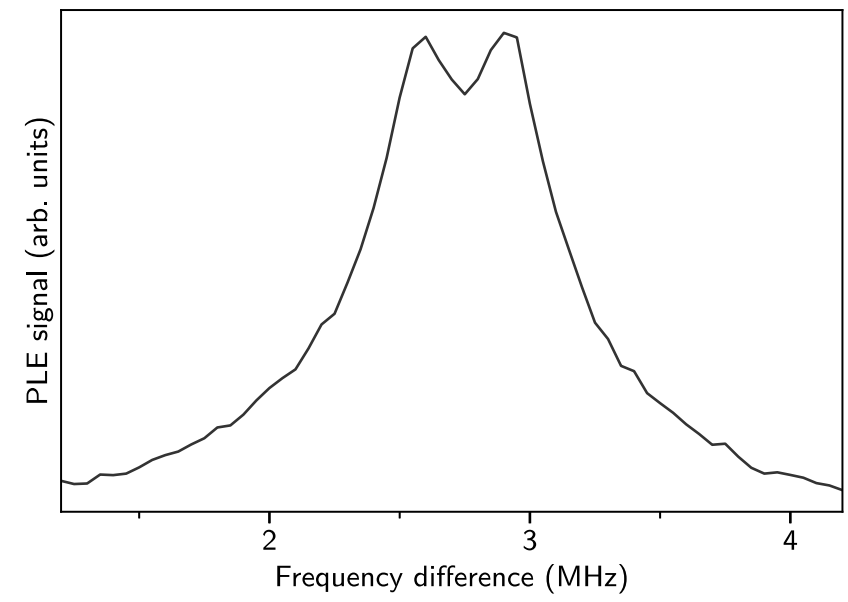

FIG. 7. ODMR response at approximately $80 \mathrm{mT}$ when pumping optical transition $B_{1}$ and symmetrically sweeping two microwave frequencies outwards from the central MW frequency under these conditions, $g_{E} \mu_{B}\left|B_{0}\right|=2.255 \mathrm{GHz}$, plotted as a function of the difference between the two swept frequencies. The observed doublet depolarization response at difference frequencies of 2.57(1) $\mathrm{MHz}$ and 2.93(1) $\mathrm{MHz}$ reveals two distinct hyperfine splittings from two slightly different orientational subsets of subset 1 , which are not optically resolvable.

the total number of orientational subsets to twelve. A twoLorentzian fit reveals effective hyperfine magnitudes $\left|A_{\text {eff }}\right|$ of 2.93(1) and 2.57(1) $\mathrm{MHz}$ for subset 1 and subset $1^{\prime}$, respectively. This has implications for the magnetic resonance experiments discussed later in this section. For the purpose of generating the green data in Fig. 6(a), a single $\left|A_{\text {eff }}\right|$ near the average of these two values is used.

Starting from the average $\left|A_{\text {eff }}\right|$ for subsets 1 and $1^{\prime}$ measured above, it is possible to refine the MW frequency estimates by fixing one MW frequency and sweeping the other. The results of this are seen in Figs. 8(a) and 8(c). In both instances, if only one MW transition is being resonantly driven, no substantial ODMR signal is observed, yet if both "allowed" MW transitions are being driven, the ODMR signal is recovered. Equivalently put, both the electron and nuclear spins can be efficiently hyperpolarized using a single optical frequency and a selectively resonant MW frequency, similar to what has been reported for excitons bound to shallow donors [47]. Interestingly, the ODMR signal could also be recovered by driving the so-called "forbidden" MW transitions, labeled $X_{M}$ (which connect the mixed spin eigenstates) and $X_{P}$ (which connect the pure spin eigenstates) in Fig. 8. Forbidden MW transitions can be driven in systems with an anisotropic hyperfine interaction [48].

Notably, the hyperpolarization mechanism for $T$ centers is not presently believed to include a substantial Auger bound exciton recombination component as is known to be the case for the shallow donors. Efforts are made at zero magnetic field to observe a change in sample conductivity

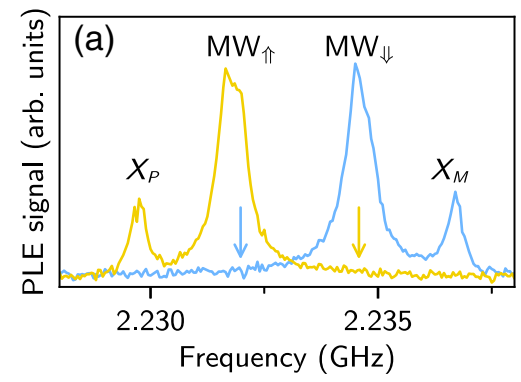

(b)
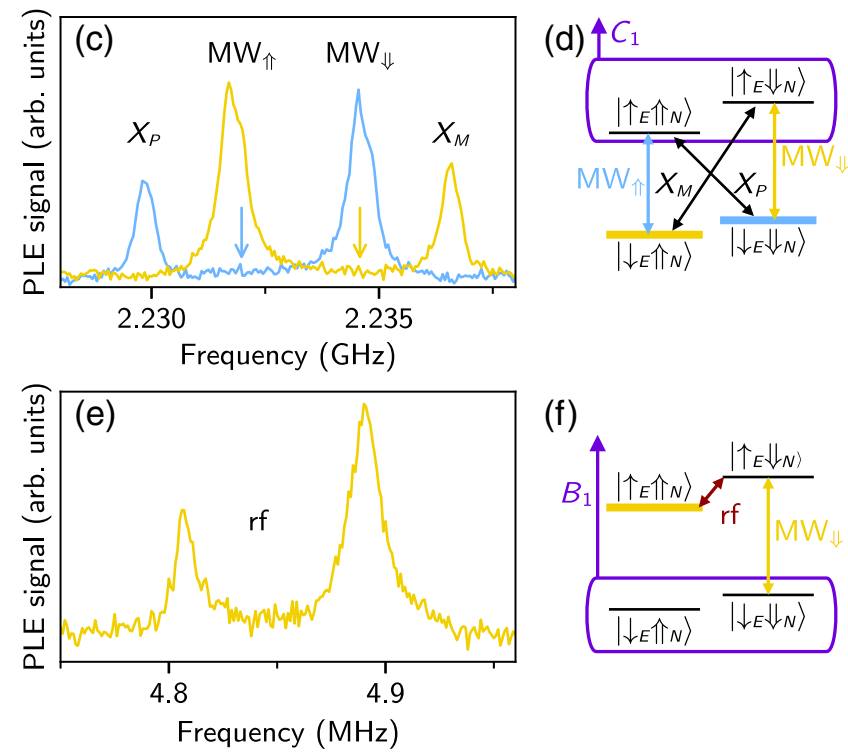

(f)

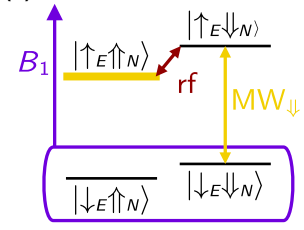

FIG. 8. ODMR peaks reflecting a relaxation of the hyperpolarization generated by pumping a fixed optical and microwave transition are observed as a second MW or rf frequency is scanned across specific resonances. (a) ODMR intensity as a second MW source is scanned while pumping optical transition $B_{1}$ as well as either $\mathrm{MW}_{\Uparrow}=2.23198 \mathrm{MHz}$ (blue data and arrow) or $\mathrm{MW}_{\Downarrow}=2.23458 \mathrm{MHz}$ (yellow data and arrow). (b) Level scheme for (a) showing the shelving level for both fixed frequencies in the absence of depolarization as a thick appropriately colored line. (c),(d) As for (a) and (b) but while pumping optical transition $C_{1}$. (e) ODMR response while scanning a rf pump frequency across the region corresponding to the $\mathrm{rf}$ transition shown in (f) while also pumping optical transition $B_{1}$ as well as $\mathrm{MW}_{\Downarrow}$.

when the laser is applied on resonance with the ZPL, as is used with great success to indirectly measure the spindependent creation of excitons bound to shallow donors [32]. For $T$ centers, these efforts did not reveal any evidence for Auger recombination whatsoever. Moreover, we have not observed any optical bleaching effects by driving the $\mathrm{TX}_{0}$ transition. Together this indicates that, with a high probability, an unpaired electron remains bound to the $T$ center following bound exciton recombination.

Starting from a spin-polarized configuration obtained by applying both on-resonant optical $B_{1}$ and MW signals, we can perform nuclear magnetic resonance (NMR) by applying radio frequency (rf) signals resonant with the nuclear spin transition frequencies. The predicted hydrogen spin 
transition frequencies near 2.1 and $4.9 \mathrm{MHz}$ correspond to the cases where the hydrogen spin is coupled to the two different electron spin states. With $\mathrm{MW}_{\Downarrow}$ continuously driven and the $B_{1}$ optical transition as chosen, which is pumping from the electron spin-down state, only the electron spin-up rf frequency should depolarize the otherwise hyperpolarized nuclear spin state and generate the PLE signal. This configuration is shown in Fig. 8(f). The sign of $A_{\text {eff }}$ determines whether the electron spin-up rf frequency is near $2.1 \mathrm{MHz}$ or near 4.9 MHz. In Fig. 8(e) we observe two rf transitions near $4.9 \mathrm{MHz}$, corresponding to the two addressable orientational subsets 1 and $1^{\prime}$ first identified in Fig. 7, which are more clearly distinguished here. From this we infer that $A_{\text {eff }}$ for these orientational subsets under these conditions are negative: $A_{\text {eff }}=-2.93(1)$ and -2.57 (1) MHz. A negative $A_{\text {eff }}$ can be observed when the anisotropic dipolar hyperfine component reaches values larger than that of the isotropic contact hyperfine component of the overall hyperfine interaction [48].

In this work the $T$ centers under investigation are those with ${ }^{12} \mathrm{C}$ constituents. Upcoming studies with ${ }^{13} \mathrm{C}$ will offer four spin $-\frac{1}{2}$ qubits per $T$ center, with a correspondingly richer spin Hamiltonian for each orientational subset.

\section{PULSED SPIN RESONANCE}

From Sec. VII we have determined spin Hamiltonian values for orientational subset 1 as well as techniques able to efficiently hyperpolarize both the electron and nuclear spin states. In this section, we introduce pulsed optical and magnetic resonance techniques that are able to manipulate and measure the $T$ center spin populations. These techniques are employed to extract spin $T_{1}$ times substantially longer than $16 \mathrm{~s}$ and Hahn-echo $T_{2}$ times exceeding a millisecond and a second for the electron and nuclear spins, respectively.

All spin measurements are predicated upon having spin initialization and readout techniques. The electron-nuclear spin hyperpolarization process outlined in Sec. VII, whereby resonant optical and MW frequencies are applied to the entire ensemble, is used to initialize the spins. Next, we discuss pulsed optical techniques to readout the $T$ center spin populations.

We begin with the electron spin polarization, which could be measured most easily by switching the optical pump signal back and forth between $B_{1}$, which pumps the system towards electron polarization +1 (all electron spins up), and $C_{1}$, which pumps the system towards electron polarization -1 (all electron spins down). In both cases the steady-state sideband luminescence approaches zero, since all systems are pumped into a shelving state that the optical pump does not access, but when switching from one pump to the other, there will be an initial luminescence transient whose amplitude is proportional to the difference in the polarization state just before the pumping is switched and the steady-state polarization that the pumping eventually produces. This scheme would require two single frequency lasers, so we used a different approach.

A mixed electron spin state (electron polarization 0 ) can be produced by applying $B_{1}, \mathrm{MW}_{\Uparrow}$, and $\mathrm{MW}_{\Downarrow}$ simultaneously, as shown in Fig. 9(b). An electron spin polarization transient is produced by shutting off $\mathrm{MW}_{\Uparrow}$ and $\mathrm{MW}_{\Downarrow}$ and letting $B_{1}$ hyperpolarize the electron spin into $|\uparrow E\rangle$, as shown in Fig. 9(c). The transient luminescence shown in Fig. 9(a) (blue data) is the signal generated when the pumping is switched from Figs. 9(b) and 9(c), or in other words the electron polarization is driven from 0 to +1 .

In principle, the electron spin state can be deduced optically during this process by detecting the resulting sideband luminescence transient. However, this electron-spin-selective optical excitation cycle leads to
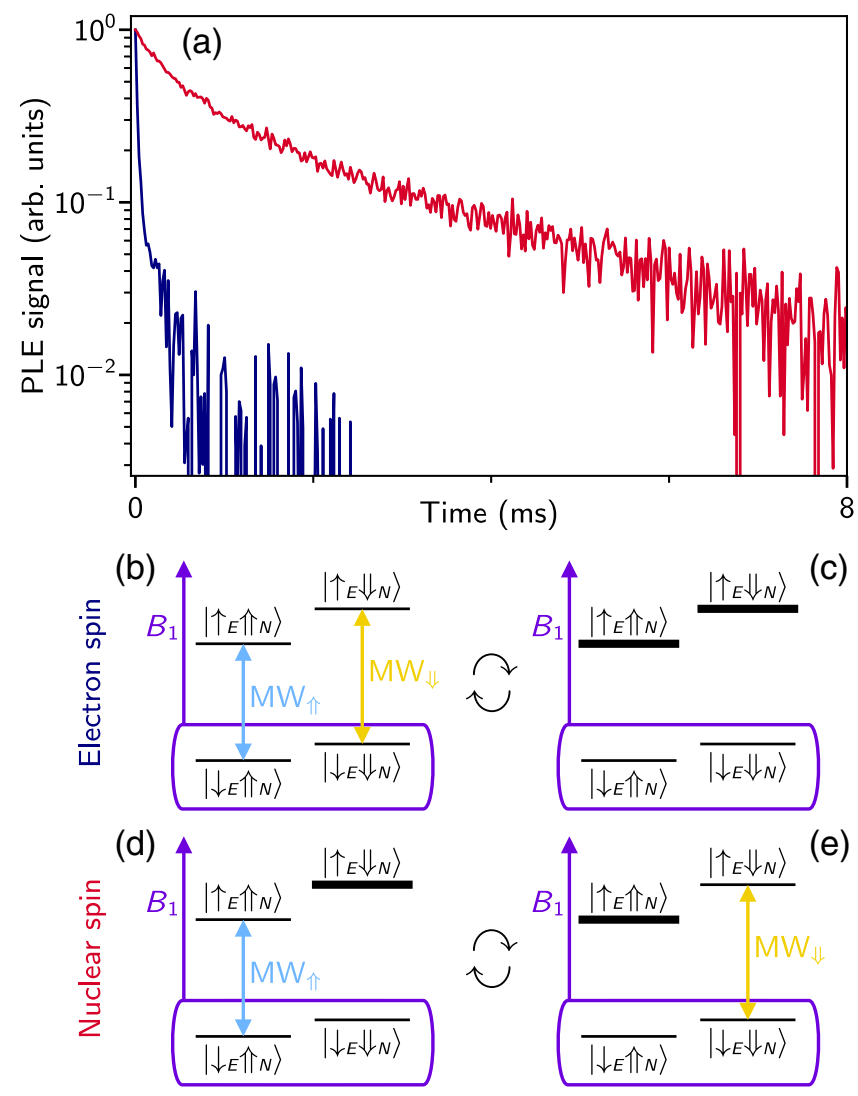

FIG. 9. (a) Nuclear spin (red) and electron spin (dark blue) polarization transients. (b),(c) Energy level diagrams illustrating the electron spin mixing (b) and polarization (c) transient schemes. (b) Populations are first prepared into a mixed population distribution by activating $B_{1}$ as well as $\mathrm{MW}_{\Uparrow}$ and $\mathrm{MW}_{\Downarrow}$. (c) To observe the electron spin polarization transient, the MW signals are halted. Shelving is indicated by a thicker energy level. (d),(e) Energy level diagrams illustrating the nuclear spin polarization transient scheme. (d) Populations are first prepared into the $\left|\uparrow_{E} \Downarrow_{N}\right\rangle$ state by pumping $B_{1}$ and $\mathrm{MW}_{\Uparrow}$. (e) To collect the transient, $\mathrm{MW}_{\Uparrow}$ is turned off while $\mathrm{MW}_{\Downarrow}$ is turned on, changing the shelving state from $\left|\uparrow_{E} \Downarrow_{N}\right\rangle$ to $\left|\uparrow_{E} \uparrow_{N}\right\rangle$. 
very rapid electron spin hyperpolarization. The branching ratios from the BE states back down to the ground electron spin states are presumably relatively balanced, and the electron spin is hyperpolarized within a few optical cycles. As a result, very few sideband luminescence photons are collected during electron spin transient measurements.

Instead of measuring the electron spin using these transients, an indirect method measuring the nuclear spin state is employed. The nuclear spin luminescence polarization transient data is shown Fig. 9(a) (red data), and the preparation and readout schemes are shown in Figs. 9(d) and 9(e), respectively. The pumping in Fig. 9(d) polarizes the system to the $\left|\uparrow_{E} \Downarrow_{N}\right\rangle$ state, while that in (e) polarizes it to the $\left|\uparrow_{E} \Uparrow_{N}\right\rangle$ state. The red luminescence transient data in Fig. 9(a) is generated when the pumping is switched from (d) to (e). A very similar transient is observed when the pumping is switched from (e) to (d).

What is noteworthy in Fig. 9(a) is the much longer decay time, and thus integrated transient area, of the nuclear polarization transient (red) compared to the electron polarization transient (blue). This must result from the fact that the $T$ centers can go through many optical absorptionemission cycles before the nuclear spin is flipped. The direct measurement of the electron polarization as in Fig. 9 has a much lower signal-to-noise ratio than the measurement of the nuclear polarization. In the following measurements the electron polarization is therefore measured indirectly, by mapping it onto the nuclear spin using an electron-spin-selective nuclear $\pi$ pulse.

Furthermore, the alternating initialization and readout cycles used in Fig. 9 can be simplified by using a single polarization combination, in our case that shown in Fig. 9(e), which leaves the system in $\left|\uparrow_{E} \Uparrow_{N}\right\rangle$, to both detect any polarization transient and then reinitialize the system. This initialization-readout procedure is labeled "POL" in Fig. 10, and the pump laser is mechanically blocked in the interval between POL cycles. If the nuclear spins do not change between one cycle and the next, there will be no transient, and if the nuclear spin state polarization differs through decay or spin manipulation, a transient will be generated whose amplitude will be proportional to the fraction of centers having nuclear spin down.

In Fig. 10(a) we combine these methods to measure Rabi oscillations when driving the nuclear spins with variable length rf pulses at the electron spin-up rf frequency. For zero rf pulse length, there is no change in nuclear spin, so there is zero transient area, and the nuclear spin polarization is near +1 , as it is at the end of the POL period. When the $\mathrm{rf}$ pulse length reaches $\pi$, the population is flipped to $\left|\uparrow_{E} \Downarrow_{N}\right\rangle$, and the nuclear polarization is near -1 .

In Fig. 10(b) we measure the Rabi oscillations due to variable length MW pulses that drive the electron spin. As before, the system is initialized to $\left|\uparrow_{E} \uparrow_{N}\right\rangle$ before the POL pumping is stopped and the spin pulse sequence is applied. The $\mathrm{MW}_{\Uparrow}$ pulse applied to this state rotates the

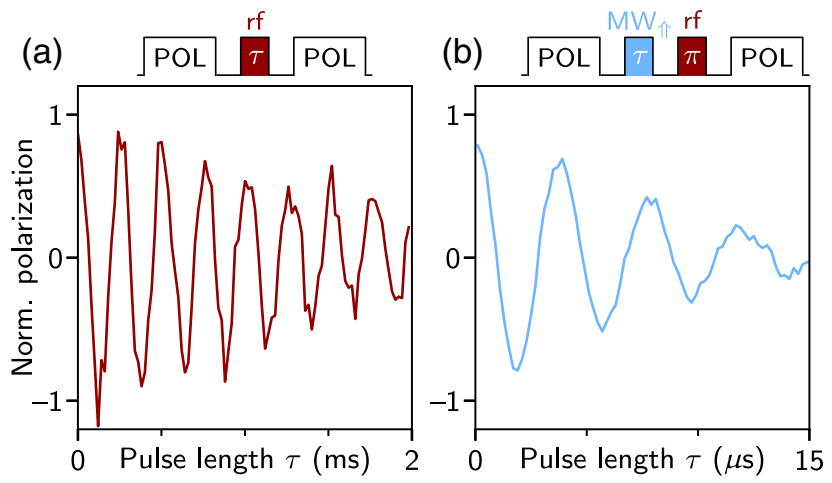

FIG. 10. Rabi oscillations for (a) the nuclear spin and (b) the electron spin. The signal decay is limited by the EPR and NMR resonators' magnetic field inhomogeneities. The normalization is determined from exponentially decaying cosine fits. The pulse sequence colors match the transition energies in the level diagrams shown in Figs. 6, 8, and 9.

electron spins, and the rf $\pi$ pulse flips any remaining population within $\left|\uparrow_{E} \Uparrow_{N}\right\rangle$ to $\left|\uparrow_{E} \Downarrow_{N}\right\rangle$, generating a nuclear polarization transient when $B_{1}$ and $\mathrm{MW}_{\Downarrow}$ are turned on again in the POL cycle. For zero $\mathrm{MW}_{\Uparrow}$ pulse length, the rf $\pi$ pulse flips all of the systems back to $\left|\uparrow_{E} \Downarrow_{N}\right\rangle$, and a maximum sideband luminescence transient area is observed, corresponding to electron spin polarization near +1 .

In Fig. 10 the normalization of the observed Rabi oscillations is determined from exponentially decaying cosine fits. The decay of the Rabi oscillations seen in Fig. 10 is thought to be due to MW and rf field inhomogeneities, and the observed spin control fidelities are typical for ensemble measurements in home-built resonators.

Pulse durations are chosen based upon these Rabi oscillations in order to extract the $T_{1}$ and Hahn-echo $T_{2}$ times of both the electron spin and hydrogen nuclear spin, with pulse sequences shown in Figs. 11(a) and 11(b). In the case of the electron spin $T_{1}$ and $T_{2}$ data, the electron spin $S_{Z}$ observable is mapped to the nuclear spin $I_{Z}$ observable for readout using a leading conditional $\pi$ pulse (a controlled NOT gate). The signal-to-noise ratio on a single shot is just above 1 in the case of the nuclear spin observable, and below 1 for the electron spin, due to imperfect pulses generated by our home-built magnetic resonance assembly. As such, averaging is required to extract accurate lifetimes and coherence times.

The resulting normalized $T_{1}$ lifetimes and $T_{2}$ Hahn-echo coherence times of the electron spin and nuclear spin are shown in Figs. 11(c) and 11(d), respectively. The $T_{1}$ times for both the electron spin $\left(T_{1 E}\right)$ and nuclear spin $\left(T_{1 N}\right)$ are far beyond their measured Hahn-echo times; we observed no signal decay out to $16 \mathrm{~s}$ for both the electron spin and nuclear spin. Averaged Hahn-echo times, extracted by fitting the averaged data to stretched exponentials, are 2.1(1) $\mathrm{ms}$ [stretch factor 4.1(7)] and 0.28(1) $\mathrm{s}$ [stretch 


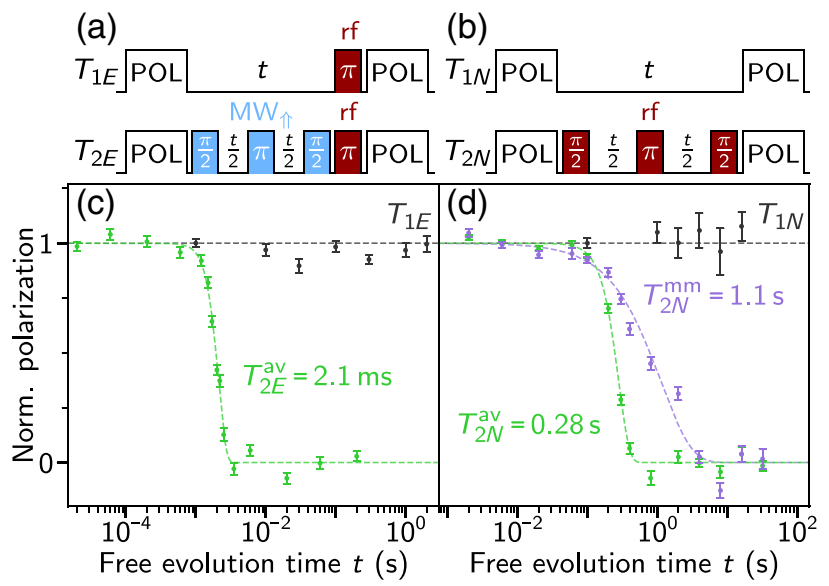

FIG. 11. (a),(b) Pulse sequences used to measure the electron spin (a) and nuclear spin (b) $T_{1}$ and $T_{2}$ times. The pulse colors match the spin transition frequencies in Fig. 1(c). The initialization and measurement POL protocols are common to all four sequences. (c),(d) Normalized $T_{1}$ and Hahn-echo $T_{2}$ decay curves for the electron (c) and hydrogen nuclear spin (d) for one orientational subset of $T$ centers. The electron spin Hahn-echo $T_{2 E}^{\mathrm{av}}$ time is limited by phase noise beyond $2 \mathrm{~ms}$. The hydrogen nuclear spin Hahn-echo $T_{2 N}^{\mathrm{av}}$ time is limited by phase noise beyond $280 \mathrm{~ms}$, which can be partially circumvented by using maximum magnitude techniques (see the text), revealing $T_{2 N}^{\mathrm{mm}}>1 \mathrm{~s}$.

factor 2.9(4)] for the electron spin $\left(T_{2 E}^{\mathrm{av}}\right)$ and nuclear spin $\left(T_{2 N}^{\mathrm{av}}\right)$, respectively. These high stretch factors are consistent with instrumental phase noise as has been observed in similar spin measurements [5]. In the case of the nuclear spin there is a sufficient signal-to-noise ratio to use maximum-magnitude techniques [5] to partially mitigate this instrumental limitation. The top $10 \%$ highest measurement values per time point are averaged to establish a tighter lower bound on the true Hahn-echo $T_{2}$, resulting in an exponential decay of $T_{2 N}^{\mathrm{mm}}=1.1(2) \mathrm{s}$ (stretch factor 1). Moving forward, dynamical decoupling [5] or clock transition [33] techniques could be employed to extract even longer coherence times.

\section{CONCLUSION}

We have reported upon the promising optical and spin properties of an ensemble of $T$ centers in ${ }^{28} \mathrm{Si}$. We have performed above-band-gap PL, resonant PL, PLE, and ODMR upon this ensemble. We have reported submicrosecond optical lifetimes in the telecommunications $O$-band near $1326 \mathrm{~nm}$, which is very close to the zero dispersion wavelength of standard silica single-mode fibers, with no observable Auger nonradiative recombination, and a Debye-Waller factor of 0.23(1). As has been reported with other radiation damage centers' ZPL transitions in ${ }^{28} \mathrm{Si}$, we observe a significant (here nearly 200 fold) reduction in ensemble inhomogeneous linewidth, to as low as 33(2) MHz at approximately 1.4 K. PLE traces at approximately $1.4 \mathrm{~K}$ confirm that this ensemble linewidth is inhomogeneously broadened. We have observed two distinct carbon isotope shifts of the ZPL, which is consistent with existing atomic models of the $T$ center. This opens up avenues for the future study of $T$ centers with up to three spin $-\frac{1}{2}$ nuclei, which may prove useful for modular quantum information processing, error detection, and quantum state purification objectives.

In a magnetic field, we have observed twelve orientational subsets, consistent with the $C_{1 h}$ symmetry of the center. The hyperpolarization dynamics observed in PLE in a magnetic field allowed for ODMR and the determination of MW and rf transition frequencies for a single orientational subset of $T$ centers. From this we have proven that the unexcited ground state contains an unpaired electron spin, conclusively resolving this open question in the literature. Furthermore, we have shown that there exists an anisotropic hyperfine interaction with the defect's hydrogen nuclear spin, suitable to support spin readout via the nuclear spin's slow hyperpolarization optical transient. Using this readout mechanism, we performed optically detected magnetic resonance upon the electron and hyperfine-coupled hydrogen nuclear spin, and extracted competitive Hahn-echo $T_{2}$ spin lifetimes for each.

Taken together these results pave the way for $T$ centers to successfully hybridize silicon's two dominant quantum platforms, and provide a long-lived multiqubit backbone for future telecom-wavelength integrated quantum photonic circuits.

\section{ACKNOWLEDGMENTS}

This work was supported by the Natural Sciences and Engineering Research Council of Canada (NSERC), the Canada Research Chairs program (CRC), the Canada Foundation for Innovation (CFI), the B.C. Knowledge Development Fund (BCKDF), and the Canadian Institute for Advanced Research (CIFAR) Quantum Information Science program. The ${ }^{28} \mathrm{Si}$ samples used in this study were prepared from the Avo 28 crystal produced by the International Avogadro Coordination (IAC) Project (2004-2011) in cooperation among the BIPM, the INRIM (Italy), the IRMM (EU), the NMIA (Australia), the NMIJ (Japan), the NPL (UK), and the PTB (Germany). We thank Valentin Karasyuk for fruitful discussions. We thank Alex English of Iotron Industries for assistance with electron irradiation.

\section{APPENDIX A: SAMPLE PREPARATION}

Unless otherwise specified, all measurements are performed upon an isotopically purified ${ }^{28} \mathrm{Si}$ crystal obtained from the Avogadro project with $99.995 \%{ }^{28} \mathrm{Si}$, less than $10^{14}$ oxygen $/ \mathrm{cm}^{3}$, and less than $5 \times 10^{14}$ carbon $/ \mathrm{cm}^{3}$ [35]. An irradiation dose of $320 \mathrm{kGy}$ is applied to the crystal using $10 \mathrm{MeV}$ electrons, with intermittent application of cooling dry ice to maintain a relatively low sample 
temperature during irradiation. To increase hydrogen concentration, the sample is first annealed in boiling water for $24 \mathrm{~h}$ [49], and then annealed in air from $300^{\circ} \mathrm{C}$ to $450{ }^{\circ} \mathrm{C}$ in steps of $30 \mathrm{~min}$ on a hotplate [25]. To reduce laser scatter at the silicon interface, a coarse polish is applied to the larger faces of the sample, followed by a brief etch in a 1:10 $\mathrm{HF} / \mathrm{HNO}_{3}$ solution to remove surface strain.

A second isotopically purified ${ }^{28} \mathrm{Si}$ crystal, with a higher carbon concentration of $1.5 \times 10^{15} \mathrm{~cm}^{-3}$, is used for the phonon sideband spectra. The same radiation and annealing treatment is applied to this sample as the first, and the TX PL signal is approximately 3-4 times stronger. Lastly, a nat $\mathrm{Si}$ sample that is lightly doped with gallium, electron irradiated, and then annealed at $500{ }^{\circ} \mathrm{C}$ in the year 1995 and stored at room temperature since then is used for the ${ }^{\text {nat }} \mathrm{Si}$ PL spectrum.

\section{APPENDIX B: METHODS}

Cryogenics.-The samples are loosely mounted in a strain-free manner and immersed in liquid helium-4 $\left(\mathrm{LHe}^{4}\right)$ at temperatures ranging from 1.4 to $4.2 \mathrm{~K}$. The temperature is set by pumping on the $\mathrm{LHe}^{4}$ bath .

Pulsed photoluminescence.-To measure the lifetime of the $T$ ZPL, $965 \mathrm{~nm}$ light pulses, at a repetition rate of $125 \mathrm{kHz}$ and with subnanosecond duration, are generated by a Picoquant PLD 800-D and directed to the sample held at a temperature of either 1.4 or $4.2 \mathrm{~K}$. The $T$ ZPL emission is filtered through a double spectrometer and routed into an ID230 single-photon detector whose output is directed to a multichannel scaler with 40 ns time resolution.

$\mathrm{CW}$ photoluminescence.-To generate nonresonant photoluminescence, the sample is illuminated with up to $300 \mathrm{~mW}$ of $1047 \mathrm{~nm}$ (beam diameter 2-4 mm) above-gap excitation. The resulting luminescence from the sample is directed into a Bruker IFS 125 HR Fourier transform infrared (FTIR) spectrometer with a $\mathrm{CaF}_{2}$ beam splitter and liquid nitrogen cooled Ge diode detector, and measured at spectral resolutions ranging from 0.25 to 62 $\mu \mathrm{eV}$. The apodization method used in shown spectra is Blackman-Harris three term, except for the $935.142 \mathrm{meV}$ ${ }^{13} \mathrm{C}$ peak doublet [Fig. 2(d)] where the boxcar (no apodization) method is used. For the magnetophotoluminescence measurements, the sample is centered in a $6 \mathrm{~T}$ superconducting magnet with the field approximately parallel to the [110] orientation.

For the resonant PL measurements, a single-frequency Toptica DL100 tunable diode laser is amplified by a Thorlabs BOA1017P amplifier to reach powers of approximately $75 \mathrm{~mW}$ (beam diameter $2-4 \mathrm{~mm}$ ), then filtered by an Edmund Optics 87-830 $1350 \mathrm{~nm}( \pm 12.5 \mathrm{~nm})$ bandpass filter and an Iridian Spectral Technologies DWDM $1329.22200 \mathrm{GHz} 1329 \mathrm{~nm}( \pm 0.5 \mathrm{~nm})$ bandpass filter before reaching the sample. Both filters are tilted down to shift their respective passbands to the laser frequency. In the optical detection path, two custom $1330 \mathrm{~nm}$ longpass filters ( $3 \mathrm{~nm}$ cut-on) from Iridian Spectral Technologies are used to filter back-reflected laser light from the sample. The resulting luminescence is directed to the FTIR spectrometer as above.

Photoluminescence excitation.-Photoluminescence excitation involves tunable resonant excitation of the $\mathrm{TX}_{0}$ ZPL followed by the optical detection of lower energy photons resulting from the TX phonon sideband. In the case of resonantly driving the $1326 \mathrm{~nm}$ ZPL transition, a single-frequency Toptica tunable diode laser DL100 is first amplified by a Thorlabs BOA1017P amplifier to reach powers of approximately $100 \mathrm{~mW}$ (beam diameter 2-4 $\mathrm{mm})$, then filtered by $1325 \mathrm{~nm}( \pm 25 \mathrm{~nm})$ bandpass filters and directed onto a polished face of the sample.

In the optical detection path, a Semrock BLP02-1319R$251319 \mathrm{~nm}$ longpass laser rejection filter is found to give good rejection of the $\mathrm{TX}_{0}$ pump photons. To remove contributions from a silicon Raman replica at $1426 \mathrm{~nm}$, a $1375 \mathrm{~nm}$ bandpass filter ( $50 \mathrm{~nm}$ bandwidth) is also in the detection path, giving rise to a spectral PLE detection window as depicted in Fig. 2(a). The resulting photons are directed to an IDQuantique ID230 high-sensitivity InGaAs photon-counting detector.

PLE is also used for excited state spectroscopy, wherein the Toptica DL100 tunable diode laser is scanned over higher energies and $\mathrm{TX}_{0} \mathrm{ZPL}$ photons are detected by replacing the aforementioned detection filters with a $\frac{3}{4} \mathrm{~m}$ focal length double monochromator set to filter out all light apart from a small window of luminescence around $1326 \mathrm{~nm}$.

Optically detected magnetic resonance.- -Unless otherwise specified, the magnetic resonance experiments are performed with the sample in an applied magnetic field of approximately $80 \mathrm{mT}$ delivered using an iron core electromagnet. The sample is mounted in the PLE setup as described above, and placed within two nested magnetic resonance resonators: a split-ring resonator $\left(f_{\text {res }}=\right.$ $2.25 \mathrm{GHz}$, bandwidth $=10 \mathrm{MHz}$ ) for electron paramagnetic resonance, and a Helmoltz coil pair $\left(f_{\text {res }}=4.8\right.$ $\mathrm{MHz}$, bandwidth $=300 \mathrm{kHz}$ ) for nuclear magnetic resonance. The radio frequency signals are generated using signal generators SRS SG384 and SRS SG386, switches ZASWA-2-50DR+ are combined using a power splitter ZB2PD-63-S+ and are amplified to up to $1 \mathrm{~W}$ of power using amplifiers ZHL-16W-43-S+ and ZHL-1-2W+ as needed. For pulse sequencing, a Spincore Pulseblaster DDSII-300 is used.

[1] D. D. Awschalom, R. Hanson, J. Wrachtrup, and B. B. Zhou, Nat. Photon. 12, 516 (2018).

[2] M. Bock, P. Eich, S. Kucera, M. Kreis, A. Lenhard, C. Becher, and J. Eschner, Nat. Commun. 9, 1998 (2018). 
[3] M. Raha, S. Chen, C. Phenicie, S. Ourari, A. Dibos, and J. Thompson, Nat. Commun. 11, 1605 (2020).

[4] J. Covey, A. Sipahigil, S. Szoke, N. Sinclair, M. Endres, and O. Painter, Phys. Rev. Appl. 11, 034044 (2019).

[5] K. Saeedi, S. Simmons, J. Z. Salvail, P. Dluhy, H. Riemann, N. V. Abrosimov, P. Becker, H.-J. Pohl, J. J. L. Morton, and M. L. W. Thewalt, Science 342, 830 (2013).

[6] K. J. Morse, R. J. S. Abraham, H. Riemann, N. V. Abrosimov, P. Becker, H.-J. Pohl, M. L. W. Thewalt, and S. Simmons, Sci. Adv. 3, e1700930 (2017).

[7] A. DeAbreu, C. Bowness, R. J. S. Abraham, A. Medvedova, K. J. Morse, H. Riemann, N. V. Abrosimov, P. Becker, H.-J. Pohl, M. L. Thewalt, and S. Simmons, Phys. Rev. Appl. 11, 044036 (2019).

[8] C. Yin, M. Rancic, G. G. De Boo, N. Stavrias, J. C. McCallum, M. J. Sellars, and S. Rogge, Nature 497, 91 (2013).

[9] L. Weiss, A. Gritsch, B. Merkel, and A. Reiserer, arXiv:2005.01775 [physics.app-ph] (2020).

[10] M. A. Hughes, H. Li, N. Theodoropoulou, and J. D. Carey, Sci. Rep. 9, 19031 (2019).

[11] A. J. Kenyon, Semicond. Sci. Technol. 20, R65 (2005).

[12] C. Chartrand, L. Bergeron, K. J. Morse, H. Riemann, N. V. Abrosimov, P. Becker, H. J. Pohl, S. Simmons, and M. L. W. Thewalt, Phys. Rev. B 98, 195201 (2018).

[13] S. Buckley, J. Chiles, A. McCaughan, G. Moody, K. Silverman, M. Stevens, R. Mirin, S. Nam, and J. Shainline, Appl. Phys. Lett. 111, 141101 (2017).

[14] C. Beaufils, W. Redjem, E. Rousseau, V. Jacques, A. Y. Kuznetsov, C. Raynaud, C. Voisin, A. Benali, T. Herzig, S. Pezzagna, J. Meijer, M. Abbarchi, and G. Cassabois, Phys. Rev. B 97, 035303 (2018).

[15] W. Redjem, A. Durand, T. Herzig, A. Benali, S. Pezzagna, J. Meijer, A. Kuznetsov, H. Nguyen, S. Cueff, J. Gérard, I. Robert-Philip, B. Gil, D. Caliste, P. Pochet, M. Abbarchi, V. Jacques, A. Dreau, and G. Cassabois, arXiv:2001.02136 [physics.app-ph] (2020).

[16] A. Tait, S. Buckley, J. Chiles, A. N. McCaughan, S. Olson, S. P. Rao, S. W. Nam, R. Mirin, and J. Shainline, J. Phys. Photonics 2, 045001 (2020).

[17] K. Lee, K. O'Donnell, J. Weber, B. Cavenett, and G. Watkins, Phys. Rev. Lett. 48, 37 (1982).

[18] N. S. Minaev and A. V. Mudryi, Phys. Status Solidi A 68, 561 (1981).

[19] E. Irion, N. Bürger, K. Thonke, and R. Sauer, J. Phys. C: Solid State Phys. 18, 5069 (1985).

[20] A. N. Safonov, E. C. Lightowlers, G. Davies, P. Leary, R. Jones, and S. Öberg, Phys. Rev. Lett. 77, 4812 (1996).

[21] P. Leary, R. Jones, and S. Öberg, Phys. Rev. B 57, 3887 (1998).

[22] G. Davies, S. Hayama, L. Murin, R. Krause-Rehberg, V. Bondarenko, A. Sengupta, C. Davia, and A. Karpenko, Phys. Rev. B 73, 165202 (2006).

[23] S. Hayama, G. Davies, J. Tan, J. Coutinho, R. Jones, and K. Itoh, Phys. Rev. B 70, 035202 (2004).

[24] A. Henry, B. Monemar, J. L. Lindstrom, T. D. Bestwick, and G. S. Oehrlein, J. Appl. Phys. 70, 5597 (1991).
[25] E. C. Lightowlers, R. C. Newman, and J. H. Tucker, Semicond. Sci. Technol. 9, 1370 (1994).

[26] J. Gower, G. Davies, E. Lightowlers, and A. Safonov, Mater. Sci. Forum 258-263, 289 (1997).

[27] A. N. Safonov and E. C. Lightowlers, Mater. Sci. Eng., B 58, 39 (1999).

[28] E. Lightowlers, L. Jeyanathan, A. Safonov, V. Higgs, and G. Davies, Mater. Sci. Eng., B 24, 144 (1994).

[29] E. Lightowlers and A. Safonov, Mater. Sci. Forum 258-263, 259 (1997).

[30] A. N. Safonov and E. C. Lightowlers, Mater. Sci. Forum 143-147, 903 (1993).

[31] D. Schmidt, B. Svensson, M. Seibt, C. Jagadish, and G. Davies, J. Appl. Phys. 88, 2309 (2000).

[32] M. Steger, K. Saeedi, M. Thewalt, J. Morton, H. Riemann, N. Abrosimov, P. Becker, and H.-J. Pohl, Science 336, 1280 (2012).

[33] G. Wolfowicz, S. Simmons, A. Tyryshkin, R. George, H. Riemann, N. Abrosimov, P. Becker, H.-J. Pohl, S. Lyon, M. Thewalt, and J. J. L. Morton, Phys. Rev. B 86, 245301 (2012).

[34] D. Karaiskaj, M. L. W. Thewalt, T. Ruf, M. Cardona, H.-J. Pohl, G. G. Deviatych, P. G. Sennikov, and H. Riemann, Phys. Rev. Lett. 86, 6010 (2001).

[35] P. Becker, H.-J. Pohl, H. Riemann, and N. Abrosimov, Phys. Status Solidi A 207, 49 (2010).

[36] G. Davies, Phys. Rep. 176, 83 (1989).

[37] E. Irion, N. Burger, K. Thonke, and R. Sauer, Phys. Rev. B. 38, 13079 (1988).

[38] K. Thonke, N. Burger, and R. Sauer, Phys. Rev. B. 32, 6720 (1985)

[39] B. C. Rose, D. Huang, Z.-H. Zhang, P. Stevenson, A. M. Tyryshkin, S. Sangtawesin, S. Srinivasan, L. Loudin, M. L. Markham, A. M. Edmonds, D. J. Twitchen, S. A. Lyon, and N. P. de Leon, Science 361, 60 (2018).

[40] J. Z. Salvail, P. Dluhy, K. Morse, M. Szech, K. Saeedi, J. Huber, H. Riemann, N. Abrosimov, P. Becker, H.-J. Pohl, and M. Thewalt, Phys. Rev. B 92, 195203 (2015).

[41] A. Stoneham, Theory of Defects in Solids: Electronic Structure of Defects in Insulators and Semiconductors (Oxford University Press, Oxford, UK, 2001).

[42] M. Cardona, T. A. Meyer, and M. L. W. Thewalt, Phys. Rev. Lett. 92, 196403 (2004).

[43] K. J. Morse, R. J. S. Abraham, D. P. Franke, N. V. Abrosimov, and M. L. Thewalt, Phys. Rev. B 93, 125207 (2016).

[44] J. Wagner and R. Sauer, Physica B+C 117-118, 113 (1983).

[45] B. Pajot, Optical Absorption of Impurities and Defects in Semiconducting Crystals: Hydrogen-Like Centres (Springer, Heidelberg, 1983), Vol. 158.

[46] A. A. Kaplyanskii, J. Phys. Colloq. 28, C4 (1967).

[47] M. Steger, T. Sekiguchi, A. Yang, K. Saeedi, M. Hayden, M. Thewalt, K. Itoh, H. Riemann, N. Abrosimov, P. Becker, and H.-J. Pohl, J. Appl. Phys. 109, 102411 (2011).

[48] A. Schweiger and G. Jeschke, Principles of Pulse Electron Paramagnetic Resonance (Oxford University Press, Oxford, UK, 2001).

[49] Y. Ohmura, K. Takahashi, H. Saitoh, T. Kon, and A. Enosawa, Phys. B 273, 228 (1999). 